\title{
Quantum quenches and Generalized Gibbs Ensemble in a Bethe Ansatz solvable lattice model of interacting bosons
}

\author{
Balázs Pozsgay ${ }^{1}$ \\ ${ }^{1}$ MTA-BME "Momentum" Statistical Field Theory Research Group \\ 1111 Budapest, Budafoki út 8, Hungary
}

September 10, 2018

\begin{abstract}
We consider quantum quenches in the so-called $q$-boson lattice model. We argue that the Generalized Eigenstate Thermalization Hypothesis holds in this model, therefore the Generalized Gibbs Ensemble (GGE) gives a valid description of the stationary states in the long time limit. For a special class of initial states (which are the pure Fock states in the local basis) we are able to provide the GGE predictions for the resulting root densities. We also give predictions for the long-time limit of certain local operators. In the $q \rightarrow \infty$ limit the calculations simplify considerably, the wave functions are given by Schur polynomials and the overlaps with the initial states can be written as simple determinants. In two cases we prove rigorously that the GGE prediction for the root density is correct. Moreover, we calculate the exact time dependence of a physical observable (the one-site Emptiness Formation Probability) for the quench starting from the state with exactly one particle per site. In the long-time limit the GGE prediction is recovered.
\end{abstract}

\section{Introduction}

The problems of equilibration and thermalization of closed quantum systems have attracted considerable interest recently ||1, 2|. One of the central questions is whether the principles of statistical physics can be derived from the unitary time evolution of the quantum system. Research in this field has been motivated partly by new experimental techniques (for example with cold atoms [3]) where an almost perfect isolation from the environment can be achieved, and therefore equilibration induced by the system itself can be studied.

Equilibration in a quantum mechanical system means that the expectation values of physical observables approach stationary values in the long time limit. Thermalization happens when these coincide with predictions obtained from a thermal ensemble. One dimensional integrable models comprise a special class of systems which possess a family of higher conserved charges in addition to the usual ones. These extra conservation laws prevent thermalization in the usual sense. Instead, it was proposed in $\mid$ that the stationary values of local observables should be described by the Generalized Gibbs Ensemble (GGE). This ensemble includes all the charges with Lagrange-multipliers fixed by the mean values of the charges in the initial state.

Since its inception the idea of the GGE has attracted considerable interest and sparked many discussions. A large body of numerical evidence for its validity was found in the lattice model of hard-core bosons 四, 5, 6, 7] (see also $\sqrt{8}$ ) and it was proven to be true in free theories or models equivalent to free fermions [9, 10, 11, 12, 13, 14, 15, 16]. However, it was found in 17] that in the interacting spin-1/2 XXZ chain the GGE (built on the strictly local charges) gives different predictions than the Quench Action (QA) method [18], which (as opposed to the GGE) is built on first principles and does not involve any assumptions or approximations. Furthermore conclusive evidence was found in a case of a specific quench 
problem in 19 that while the predictions of the QA method coincide with results of real-time simulations, the GGE predictions [20, 21, 22] are not correct.

It was argued in [6] that equilibration to the GGE predictions can be explained by the Generalized Eigenstate Thermalization Hypothesis (GETH), which roughly states that if there are two states which have almost the same mean values of the conserved charges, then local correlations in the two states should be also close to each other. If the GETH holds than the GGE is valid for quenches from any initial state satisfying the cluster decomposition principle. It was recently shown in [23] that the failure of the GGE in the XXZ spin chain can be attributed to the failure of the GETH. It was argued in [23, 24 that this is a generic property of integrable models with multiple particle species.

The question remains whether the GETH and the GGE can be correct in any genuinely interacting integrable model. To find an example one should certainly look for models with one particle type. An obvious choice would be the Lieb-Liniger (LL) model, which is a continuum theory of 1D interacting bosons $[25]$. Quenches in the LL model have been investigated in a number of papers recently $[26,27,28,29,30,31,32,33,34$. However, it was found in |29| that for an interaction quench from zero to finite coupling the expectation values of the higher charges are divergent, and therefore the GGE can not be defined in that case. The problem was circumvented by applying a lattice regularization using the so-called $q$-boson model [35, 36, 37]. The QA solution of this interaction quench was later given in [31], where it was argued that the GGE can not be correct in the LL model due to the aforementioned divergences and the observed logarithmic singularities in the Bethe root densities, which can not be captured by the GGE.

In this paper we investigate quantum quenches in the $q$-boson model, without the goal of taking the continuum limit towards the Bose gas. In this lattice model there is only one particle type in the spectrum, and the infinities encountered in the LL model do not appear here. Therefore it is an ideal testing ground for the GETH and the GGE.

The paper is organized as follows. In Section 2 we review the Bethe Ansatz solution of the model and the construction of the higher conserved charges. In 3 we construct the GGE density matrix for this model and argue that the GETH holds, therefore the GGE is valid. In 6 we consider specific quench problems and provide the GGE predictions for a class of initial states. The $q \rightarrow \infty$ limit of the model is investigated in , where the equilibrium properties of the model are established. Quenches in the $q \rightarrow \infty$ limit are investigated in 6 , where we confirm the GGE predictions in two simple cases by analyzing the exact overlaps. Also, we derive an analytic formula for the time dependence of a simple physical observable, the one-site Emptiness Formation Probability. The long-time limit of this quantity is found to agree with the GGE prediction. The $q=1$ limit of the model (the case of free bosons) is considered in 7. Finally, Section 8 includes our conclusions, a number of remarks about our results, and a list of open problems.

\section{The model and its Bethe Ansatz solution}

Consider a lattice consisting of $L$ sites such that the configuration space of each site is a single bosonic space. Let us define the canonical Bose operators $b_{j}, b_{j}^{\dagger}, N_{j}$ acting on site $j$ by the usual commutation relations

$$
\left[b_{j}, b_{k}^{\dagger}\right]=\delta_{j, k} N_{k} \quad\left[N_{j}, b_{k}\right]=-\delta_{j, k} b_{k} \quad\left[N_{j}, b_{k}^{\dagger}\right]=-\delta_{j, k} b_{k}^{\dagger} .
$$

The action of these operators on the local states $|n\rangle_{j}, n=0 \ldots \infty$ is given by

$$
b_{j}|n\rangle_{j}=\sqrt{n}|n-1\rangle_{j} \quad b_{j}^{\dagger}|n\rangle_{j}=\sqrt{n+1}|n+1\rangle_{j} \quad N_{j}|n\rangle_{j}=n|n-1\rangle_{j} .
$$

We also define the operators $B_{j}^{\dagger}, B_{j}$ by their action

$$
B_{j}|n\rangle_{j}=\sqrt{[n]_{q}}|n-1\rangle_{j} \quad B_{j}^{\dagger}|n\rangle_{j}=\sqrt{[n+1]_{q}}|n+1\rangle_{j},
$$

where

$$
[x]_{q}=\frac{1-q^{-2 x}}{1-q^{-2}}
$$


The parameter $q$ is an arbitrary real number. In the present work we will consider the cases $q \geq 1$ and we will use the parametrization $q=e^{\eta}, \eta>0$. It is easy to check that the following commutation relations hold:

$$
\left[N_{k}, B_{k}\right]=-B_{k} \quad\left[N_{k}, B_{k}^{\dagger}\right]=-B_{k}^{\dagger} \quad\left[B_{k}, B_{k}^{\dagger}\right]=q^{-2 N_{k}}
$$

These equations are the defining relations of the so-called $q$-boson algebra [38]. The canonical Bose operators are recovered in the $q \rightarrow 1$ limit:

$$
\lim _{q \rightarrow 1} B_{k}=b_{k} \quad \lim _{q \rightarrow 1} B_{k}^{\dagger}=b_{k}^{\dagger} .
$$

The $q$-boson Hamiltonian is defined as

$$
H=-\sum_{j=1}^{L}\left(B_{j}^{\dagger} B_{j+1}+B_{j+1}^{\dagger} B_{j}-2 N_{j}\right)
$$

where periodic boundary conditions are assumed. Even though the Hamiltonian (2.1) has the form of a free hopping model, there are interactions between the particles due to the fact that the $B$ and $B^{\dagger}$ are not the canonical Bose operators and the hopping amplitudes depend on the local occupation numbers. The model can serve as a lattice regularization of the Lieb-Liniger model [35, 36, 37, 39, 29]; however, in the present work we focus on the lattice model only.

The $q$-boson Hamiltonian was solved in [35] by the Algebraic Bethe Ansatz (ABA). The coordinate Bethe Ansatz wave functions were later calculated in $\mid 40$; they are given by Hall-Littlewood functions. Here we review the (ABA) solution, our exposition follows that of [39].

Let us consider an auxiliary space $V=\mathbb{C}^{2}$ and define the so-called Lax operator, which is a matrix in auxiliary space with matrix elements being operators in the bosonic Fock spaces:

$$
L(\lambda)=\left(\begin{array}{cc}
e^{\lambda} & \chi B^{\dagger} \\
\chi B & e^{-\lambda}
\end{array}\right)
$$

Here $\lambda$ is the rapidity parameter and $\chi^{2}=1-q^{-2}$. The Lax operator satisfies the YangBaxter equation

$$
R(\lambda-\mu)(L(\lambda) \otimes L(\mu))=(L(\mu) \otimes L(\lambda)) R(\lambda-\mu)
$$

with the $R$-matrix

$$
R(u)=\left(\begin{array}{cccc}
\sinh (u+\eta) & 0 & 0 & 0 \\
0 & \sinh (\eta) & q \sinh (u) & 0 \\
0 & q^{-1} \sinh (u) & \sinh (\eta) & 0 \\
0 & 0 & 0 & \sinh (u+\eta)
\end{array}\right)
$$

The central object of the ABA is the monodromy matrix, which is given by

$$
T(\lambda)=L_{L}(\lambda) L_{L-1}(\lambda) \ldots L_{1}(\lambda)=\left(\begin{array}{ll}
A(\lambda) & B(\lambda) \\
C(\lambda) & D(\lambda)
\end{array}\right) .
$$

It follows from (2.2) that the monodromy matrix satisfies the RTT-relation

$$
R(\lambda-\mu)(T(\lambda) \otimes T(\mu))=(T(\mu) \otimes T(\lambda)) R(\lambda-\mu) .
$$

A direct consequence of (2.4) is that the transfer matrix defined as

$$
\tau(\lambda)=\operatorname{Tr} T(\lambda)=A(\lambda)+D(\lambda)
$$

satisfies

$$
[\tau(\lambda), \tau(\mu)]=0
$$

It is easy to see that

$$
\lim _{\lambda \rightarrow-\infty}\left(e^{L \lambda} \tau(\lambda)\right)=1
$$


The properties (2.5) and (2.6) enable us to obtain a commuting set of local charges by expanding the logarithm of the transfer matrix around the point $\lambda=-\infty$. We define

$$
I_{m}=\left.\frac{1}{(2 m) !}\left(\frac{\partial}{\partial \xi}\right)^{2 m} \log \left(\xi^{L} \tau(\xi)\right)\right|_{\xi=0},
$$

where $\xi=e^{\lambda}$. It follows from the definition of the transfer matrix and the form of the Lax operator that $I_{m}$ is a sum of local operators which span at most $m+1$ sites. The first two examples are

$$
\begin{aligned}
& I_{1}=\chi^{2} \sum_{j} B_{j}^{\dagger} B_{j+1} \\
& I_{2}=\chi^{2}\left(1-\frac{\chi^{2}}{2}\right) \sum_{j}\left(B_{j}^{\dagger} B_{j+2}-\frac{\chi^{2}}{2-\chi^{2}} B_{j}^{\dagger} B_{j}^{\dagger} B_{j+1} B_{j+1}-\chi^{2} B_{j}^{\dagger} B_{j+1}^{\dagger} B_{j+1} B_{j+2}\right) .
\end{aligned}
$$

A formula for $I_{3}$ is also given in [39].

These operators are not Hermitian. It is useful to define the charges with negative indices as their adjoint:

$$
I_{-n}=\left(I_{n}\right)^{\dagger} .
$$

They can be obtained by expanding the transfer matrix around $\lambda=\infty$.

The particle number operator

$$
N=\sum_{j} N_{j}
$$

commutes with all of the charges, which follows from the fact that the transfer matrix only includes terms with an equal number of $B^{\dagger}$ and $B$ operators. We define $I_{0} \equiv N$. The Hamiltonian can then be written as

$$
H=-\frac{I_{1}+I_{-1}}{\chi^{2}}+2 I_{0} .
$$

Eigenstates of the system are constructed using the $B$-operators of the monodromy matrix:

$$
\left|\{\lambda\}_{N}\right\rangle=\prod_{j=1}^{N} B\left(\lambda_{j}\right)|0\rangle,
$$

where $|0\rangle$ is the Fock vacuum. The parameters $\lambda_{j}$ are the rapidities of the interacting bosons. A state of the form (2.9) is an eigenstate of the transfer matrix if the rapidities satisfy the Bethe equations:

$$
e^{2 L \lambda_{j}} \prod_{k \neq j} \frac{\sinh \left(\lambda_{j}-\lambda_{k}+\eta\right)}{\sinh \left(\lambda_{j}-\lambda_{k}-\eta\right)}=1 .
$$

The eigenvalues of the transfer matrix on the Bethe states are

$$
\tau(u)\left|\{\lambda\}_{N}\right\rangle=\frac{1}{q^{N}}\left(e^{L u} \prod_{j=1}^{N} f\left(u, \lambda_{j}\right)+e^{-L u} \prod_{j=1}^{N} f\left(\lambda_{j}, u\right)\right)\left|\{\lambda\}_{N}\right\rangle,
$$

where

$$
f(u)=\frac{\sinh (u+\eta)}{\sinh (u)}
$$

Eigenvalues of the local charges are easily obtained using the definition (2.7). It is easy to see that they can be expressed as sums of single particle eigenfunctions:

$$
I_{m}\left|\{\lambda\}_{N}\right\rangle=\sum_{j=1}^{N} i_{m}\left(\lambda_{j}\right)
$$

where

$$
i_{m}(\lambda)=\left.\frac{1}{(2 m) !}\left(\frac{\partial}{\partial \xi}\right)^{2 m} \log (f(\lambda, \log (\xi)))\right|_{\xi=0}
$$


In (2.12) we used that the charge $I_{m}$ only exists in lattices with $L>m$, therefore it is enough to keep the second term from (2.11). Using the substitution $e^{\lambda}=a$ the derivatives are calculated easily:

$$
\begin{aligned}
i_{m}(\lambda) & =\left.\frac{1}{(2 m) !}\left(\frac{\partial}{\partial \xi}\right)^{2 m}\left[\log \left(1-\xi^{2} /\left(a^{2} q^{2}\right)\right)-\log \left(1-\xi^{2} / a^{2}\right)\right]\right|_{\xi=0} \\
& =\frac{1}{m}\left(-\frac{1}{(a q)^{2 m}}+\frac{1}{a^{2 m}}\right)=\frac{1}{m}\left(1-q^{-2 m}\right) e^{-2 m \lambda}
\end{aligned}
$$

It is useful to parametrize the rapidities as $\lambda=i p / 2$. This way the Bethe equations take the form

$$
e^{i p_{j} L} \prod_{k \neq j} \frac{\sin \left(\left(p_{j}-p_{k}\right) / 2-i \eta\right)}{\sin \left(\left(p_{j}-p_{k}\right) / 2+i \eta\right)}=1 .
$$

In the case of $\eta>0$ considered in the present work all solutions to (2.13) are real numbers and they can be chosen to lie in the interval $[-\pi, \pi]$.

In terms of the $p$-variables the single particle eigenvalues of the charges take the form

$$
i_{m}(p)=\frac{1}{|m|}\left(1-q^{-2|m|}\right) e^{-i m p}
$$

The single particle energy is

$$
e(p)=4 \sin ^{2}(p / 2) .
$$

The $p$-variables are the physical pseudo-momenta on the lattice, because the single particle eigenvalue for the translation by one-site is $e^{i p}$.

For the sake of completeness we note that the local $q$-boson operators at sites 1 and $L$ can be reconstructed from the off-diagonal elements of the monodromy matrix as

$$
\begin{array}{ll}
\lim _{\lambda \rightarrow \infty}\left(e^{-(L-1) \lambda} B(\lambda)\right)=\chi B_{1}^{\dagger} & \lim _{\lambda \rightarrow-\infty}\left(e^{(L-1) \lambda} B(\lambda)\right)=\chi B_{M}^{\dagger} \\
\lim _{\lambda \rightarrow \infty}\left(e^{-(L-1) \lambda} C(\lambda)\right)=\chi B_{M} & \lim _{\lambda \rightarrow-\infty}\left(e^{(L-1) \lambda} C(\lambda)\right)=\chi B_{1} .
\end{array}
$$

However, there are no such formulas for the other local $q$-boson operators and the general solution of the so-called "quantum inverse problem" 41, 42] is not known.

\section{$2.1 \quad$ Thermodynamic limit}

We will be interested in physical situations where there is a large number of particles in a large volume such that the particle density is finite. As usually we introduce the densities of Bethe roots $\rho_{r}(p)$ and holes $\rho_{h}(p)$ such that in a large volume the total particle density is given by

$$
\frac{N}{L}=\int_{-\pi}^{\pi} \frac{d p}{2 \pi} \rho_{r}(p)
$$

It follows from the Bethe equations that

$$
\rho_{r}(p)+\rho_{h}(p)=1+\int \frac{d u}{2 \pi} \varphi(p-u) \rho_{r}(u)
$$

with

$$
\varphi(u)=\frac{\sinh (2 \eta)}{\cosh (2 \eta)-\cos (u)} .
$$

Expectation values of the charges in the thermodynamic limit are then calculated as

$$
\frac{\left\langle I_{m}\right\rangle}{L}=\int_{-\pi}^{\pi} \frac{d p}{2 \pi} \rho_{r}(p) i_{m}(p)=\frac{1-q^{-2|m|}}{|m|} \int_{-\pi}^{\pi} \frac{d p}{2 \pi} \rho_{r}(p) e^{-i m p} .
$$

It is a special property of this model that the local charges measure the Fourier components of the root distribution. 


\section{Quantum quenches and the Generalized Gibbs Ensem- ble in the $q$-boson model}

We are interested in non-equilibrium situations in the $q$-boson model. We assume that at $t=0$ the state is prepared in the initial state

$$
|\Psi(t=0)\rangle=\left|\Psi_{0}\right\rangle
$$

which is not an eigenstate of the Hamiltonian. It can be the ground state of a local Hamiltonian, or any other state prepared according to certain rules. Examples will be given in Section 1 .

The time evolution of physical observables is given by

$$
\langle\mathcal{O}(t)\rangle=\left\langle\Psi_{0}\left|e^{i H t} \mathcal{O} e^{-i H t}\right| \Psi_{0}\right\rangle .
$$

We are interested in the large time behaviour of the observables in the thermodynamic limit. Neglecting degeneracies in the spectrum the long-time average in a finite volume case can be written as

$$
\lim _{T \rightarrow \infty} \int_{0}^{T} d t\langle\mathcal{O}(t)\rangle=\sum_{n}\left|c_{n}\right|^{2}\langle n|\mathcal{O}| n\rangle, \quad c_{n}=\left\langle n \mid \Psi_{0}\right\rangle .
$$

The sum over eigenstates on the r.h.s. above can be interpreted as a statistical physical ensemble, and it is called the Diagonal Ensemble (DE). The weights of the DE are given by the squared overlaps with the initial state, and it is an important question whether the predictions of the DE coincide with those of a statistical physical ensemble, at least in the thermodynamic limit. The system thermalizes, if the DE gives the same mean values as a canonical or grand-canonical Gibbs Ensemble (GE). This is the expected behaviour for a generic non-integrable model.

The situation is different in integrable models, where the existence of the higher conserved charges prevents thermalization. It was suggested in $\mid$ || that in these models the DE predictions should agree with those of a Generalized Gibbs Ensemble (GGE) which includes all higher charges.

In the case of the $q$-boson model the GGE density matrix can be defined as

$$
\rho_{G G E}=\frac{\exp \left(-\sum_{j=-\infty}^{\infty} \beta_{j} I_{j}\right)}{\operatorname{Tr} \exp \left(-\sum_{j=-\infty}^{\infty} \beta_{j} I_{j}\right)},
$$

where the Lagrange-multipliers are fixed by the requirement

$$
\left\langle\Psi_{0}\left|I_{j}\right| \Psi_{0}\right\rangle=\operatorname{Tr}\left(\rho_{G G E} I_{j}\right) .
$$

We also require $\beta_{j}=\beta_{-j}^{*}$, such that $\rho_{G G E}$ is Hermitian. This condition is consistent with (3.3).

The GGE hypothesis states that for any local operator

$$
\lim _{T \rightarrow \infty} \int_{0}^{T} d t\langle\mathcal{O}(t)\rangle=\operatorname{Tr}\left(\rho_{G G E} \mathcal{O}\right) .
$$

Time averaging is only required in finite volume, and it can be omitted in the infinite volume limit.

The GGE was proven to be correct for free theories or models equivalent to free fermions 迆, 6. 6, 7, 8, 13, 14, 43, 44, 16], but counterexamples were found in 117, 19] in the case of the XXZ spin chain. It is open question whether the GGE holds in other interacting models, and what the precise conditions are for its validity.

In [6] it was proposed that the GGE is valid whenever the Generalized Eigenstate Thermalization Hypothesis (GETH) holds. This hypothesis states that the mean values local operators in the excited states only depend on the mean values of the charges. In other words, if two excited states have mean values of the charges that are close to each other, then all local correlations in the two states will be close as well. Given that the initial state 
satisfies the cluster decomposition principle, the Diagonal Ensemble (3.1) will be populated by states which have the same conserved charges as the initial state, such that the mean deviation for the densities of the charges becomes zero in the thermodynamic limit [6, 23]. On the other hand, the GGE density matrix (3.2) produces states with the prescribed charges by definition. Therefore, if the GETH holds then the two ensembles give the same results for the local operators, because the dominating states of both ensembles will have the same local correlations. This is the reason why the validity of the GGE follows from the GETH [6]. We stress that the weights $\left|c_{n}\right|^{2}$ of the DE need not be directly related to the generalized Boltzmann-weights of the GGE.

We now argue that the GETH is valid in the repulsive $q$-boson model. In this model there is only one particle type in the spectrum, and the density of Bethe roots is described by a single function $\rho_{r}(p)$. According to 2.17) the conserved charges measure the Fourier components of $\rho_{r}(p)$ and the root density can be reconstructed from the charges as

$$
\rho_{r}(p)=\frac{1}{L}\left(\langle N\rangle+\sum_{j=1}^{\infty} \frac{j\left(e^{i j p}\left\langle I_{j}\right\rangle+e^{-i j p}\left\langle I_{-j}\right\rangle\right)}{1-q^{-2 j}}\right) .
$$

The charges thus uniquely determine the root density, and in order to prove the GETH we need to show that in the thermodynamic limit the local correlators only depend on $\rho_{r}(p)$. We are not able to prove this statement in full generality, but experience with other Bethe Ansatz solvable models suggests that it is in fact true. In subsection 3.1 we prove it for a class of non-trivial local operators.

With this we have established that the GETH holds in the $q$-boson model. As a consequence, the GGE should also hold for any initial state satisfying the cluster decomposition principle.

Equation (3.4) shows that the charges uniquely determine the root density and there is no need to obtain explicit expressions for the Lagrange-multipliers entering the GGE density matrix. However, for the sake completeness we show how to compute them.

The standard Thermodynamic Bethe Ansatz treatment of the GGE density matrix (3.2) leads to the generalized TBA equations 45 :

$$
\varepsilon(p)=\beta_{0}+\sum_{m=1}^{\infty} \frac{1-q^{-2 m}}{m}\left(\beta_{m} e^{-i m p}+\beta_{m}^{*} e^{i m p}\right)-\int_{-\pi}^{\pi} \frac{d u}{2 \pi} \varphi(p-u) \log \left(1+e^{-\varepsilon(u)}\right),
$$

where $\varepsilon(p)$ is the pseudoenergy defined as

$$
e^{\varepsilon(p)}=\frac{\rho_{h}(p)}{\rho_{r}(p)}
$$

After the root density is obtained directly from (3.4), the hole density can be calculated from (2.15). Substituting both functions into the equation

$$
\begin{aligned}
\beta_{0}+\sum_{m=1}^{\infty} \frac{1-q^{-2 m}}{m} & \left(\beta_{m} e^{-i m p}+\beta_{m}^{*} e^{i m p}\right)= \\
& \log \left(\frac{\rho_{h}(p)}{\rho_{r}(p)}\right)+\int_{-\pi}^{\pi} \frac{d u}{2 \pi} \varphi(p-u) \log \left(\frac{\rho_{r}(p)+\rho_{h}(p)}{\rho_{h}(p)}\right),
\end{aligned}
$$

the Lagrange-multipliers are obtained simply by Fourier transformation. Note that all Lagrangemultipliers have a finite well-defined value.

We wish to remark that on the lattice all charges have finite mean values, therefore the problem of infinities encountered for interaction quenches in the continuum Bose gas [29, 31 ] does not occur in the $q$-boson model. Also, equation (3.4) holds even if there are logarithmic singularities in the root density, because such functions are still members of $L^{2}([-\pi, \pi])$ and therefore their Fourier series is well defined and converges almost everywhere. Logarithmic singularities were encountered earlier in other models [31, 17], but in the $q$-boson model they do not obstruct the validity of the GGE.

In the following subsection we show how to compute the GGE predictions for a set of simple local observables. Specific quench problems are considered in Section 1 . 


\subsection{Local correlators in the GGE}

For the $q$-boson model there are no results in the literature for the excited state mean values of short range correlation functions. Here we apply the Hellmann-Feynman theorem 46] to compute mean values of certain local operators in states with arbitrary root density $\rho_{r}(p)$. The GGE predictions are then calculated by substituting the root density obtained from (3.4) into the results presented below. The method we apply was previously developed independently in $47 \mid$ and $[17]$.

As a first example consider the Hermitian operator

$$
J_{1}=B_{1} B_{2}^{\dagger}+B_{1}^{\dagger} B_{2},
$$

which is the operator density for $I_{1}+I_{-1}$. The finite volume mean values in an arbitrary Bethe state are

$$
\left\langle\Psi\left|J_{1}\right| \Psi\right\rangle=\frac{1}{L}\left(1-q^{-2}\right) \sum_{j=1}^{N} 2 \cos \left(p_{j}\right)
$$

We use the Hellmann-Feynman theorem to obtain the mean values of the operator

$$
J_{1}^{\prime}=\frac{\partial}{\partial \eta}\left(B_{1} B_{2}^{\dagger}+B_{1}^{\dagger} B_{2}\right)=E_{1} B_{2}^{\dagger}+E_{1}^{\dagger} B_{2}+B_{1} E_{2}^{\dagger}+B_{1}^{\dagger} E_{2},
$$

where $E^{\dagger}=\partial B^{\dagger} / \partial \eta$ and $E=\partial B / \partial \eta$ are defined by their action on the Fock states:

$$
E_{j}|n\rangle_{j}=\sqrt{[n]_{q}^{\prime}}|n-1\rangle_{j} \quad E_{j}^{\dagger}|n\rangle_{j}=\sqrt{[n+1]_{q}^{\prime}}|n+1\rangle_{j},
$$

where

$$
[x]_{q}^{\prime}=\frac{\partial}{\partial \eta}[x]_{q}=\frac{2 x q^{-2 x}}{1-q^{-2}}+\frac{2\left(1-q^{-2 x}\right) q^{-2}}{\left(1-q^{-2}\right)^{2}} .
$$

Taking the derivative of (3.8) with respect to $\eta=\log (q)$ leads to

$$
\left\langle\Psi\left|J_{1}^{\prime}\right| \Psi\right\rangle=\frac{1}{L} 2 q^{-2} \sum_{j=1}^{N} 2 \cos \left(p_{j}\right)+\frac{1}{L}\left(1-q^{-2}\right) \sum_{j=1}^{N}\left(-2 \sin \left(p_{j}\right)\right) \frac{d p_{j}}{d \eta} .
$$

The derivatives $\frac{d p_{j}}{d \eta}$ can be obtained from the logarithmic form of the Bethe equations:

$$
p_{j} l+\sum_{k \neq j}-i \log \frac{\sin \left(\left(p_{j}-p_{k}\right) / 2-i \eta\right)}{\sin \left(\left(p_{j}-p_{k}\right) / 2+i \eta\right)}=2 \pi I_{j}
$$

The quantum numbers $I_{j} \in \mathbb{Z}$ specify the state and can not change as we vary $\eta$, therefore

$$
L \frac{d p_{j}}{d \eta}+\sum_{k \neq j} \varphi\left(p_{j}-p_{k}\right)\left(\frac{d p_{j}}{d \eta}-\frac{d p_{k}}{d \eta}\right)+\sum_{k \neq j} \tilde{\varphi}\left(p_{j}-p_{k}\right)=0
$$

where $\varphi(p)$ is given by 2.16$)$ and

$$
\tilde{\varphi}(p)=-\frac{2 \sin (p)}{\cosh (2 \eta)-\cos (p)} .
$$

In a large volume $\frac{d p_{j}}{d \eta} \approx f\left(p_{j}\right)$, where $f(p)$ is the so-called shift function. It follows from (3.10) that it satisfies

$$
f(p)+\int \frac{d u}{2 \pi} \varphi(p-u) \rho_{r}(u)(f(p)-f(u))+\int \frac{d u}{2 \pi} \rho_{r}(u) \tilde{\varphi}(p-u)=0 .
$$

Using (2.15) we obtain

$$
f(p)\left(\rho_{r}(p)+\rho_{h}(p)\right)-\int \frac{d u}{2 \pi} \varphi(p-u) \rho_{r}(u) f(u)+\int \frac{d u}{2 \pi} \rho_{r}(u) \tilde{\varphi}(p-u)=0 .
$$


This linear equation uniquely determines $f(p)$.

Finally, the mean value of $J_{1}^{\prime}$ is expressed as

$$
\left\langle\Psi\left|J_{1}^{\prime}\right| \Psi\right\rangle=4 q^{-2} \int \frac{d p}{2 \pi} \cos (p) \rho_{r}(p)-2\left(1-q^{-2}\right) \int \frac{d p}{2 \pi} \sin (p) f(p) \rho_{r}(p) .
$$

Completely analogous results hold if we apply the Hellmann-Feynman theorem to the remaining higher charges. Defining $J_{m}$ to be the operator density of the Hermitian combination $I_{m}+I_{-m}$ we obtain

$$
\left\langle\Psi\left|J_{m}^{\prime}\right| \Psi\right\rangle=4 q^{-2 m} \int \frac{d p}{2 \pi} \cos (m p) \rho_{r}(p)-2 \frac{1-q^{-2 m}}{m} \int \frac{d p}{2 \pi} \sin (m p) f(p) \rho_{r}(p),
$$

where $J_{m}^{\prime}=\frac{\partial}{\partial \eta} J_{m}$ and the shift function is given by the solution of (3.11). We refrain from writing down the $J_{m}^{\prime}$ in terms of local operators, as they are easily obtained from the expressions of the charges $I_{m}$ ?. We just stress that all $J_{m}^{\prime}$ are non-trivial local operators which span at most $m+1$ sites.

\section{GGE for a class of initial states}

In this section we derive the GGE solution for a special class of initial states, which are given as tensor products of one-site particle number eigenstates. Translationally invariant cases are considered in 4.1, whereas 4.2 deals with states that break the translational invariance.

\subsection{Translationally invariant cases}

We consider the states $\left|F_{n}\right\rangle$ in which there is exactly $n$ particle at each site:

$$
\left|F_{n}\right\rangle=\otimes_{j=1}^{L}|n\rangle_{j}, \quad n>0 .
$$

They are not eigenstates of the $q$-boson Hamiltonian. They can be considered as ferromagnetic states pointing in a certain direction in the infinite dimensional Fock space. Also, they are the ground states of the infinitely repulsing Bose-Hubbard model at a given integer filling. The physically most relevant case is $\left|F_{1}\right\rangle$ which is a state of uniform particle density 1 . Quantum quenches in the Bose-Hubbard model with initial state $\left|F_{1}\right\rangle$ were studied in $44 \mid$.

In the following we evaluate the $q$-boson GGE predictions for quenches starting from the $\left|\Psi_{0}\right\rangle=\left|F_{n}\right\rangle$. First we compute the expectation values of the charges, then we reconstruct $\rho(p)$ from (3.4), and finally we give predictions for the local operators introduced in the previous section.

It is easy to see that all charges $I_{m}, m>1$ are built from local operators which have the form

$$
\left(B_{j_{1}}^{\dagger}\right)^{n_{1}} \ldots\left(B_{j_{2}}\right)^{n_{2}}
$$

such that $j_{2}>j_{1}, n_{1}, n_{2}>0$ and the dots stand for operators acting on sites $j_{k}$ with $j_{2}>$ $j_{k}>j_{1}$. In other words, the leftmost and rightmost operators are "unpaired": a combination of the form $B_{j_{k}}^{\dagger} B_{j_{k}}$ can only occur in the middle, but never on the two ends of the operator product. This follows simply from the definition of the transfer matrix (2.3): regarded as a power series in $e^{-\lambda}$ the transfer matrix has terms of the form

$$
\left(B_{j_{1}}^{\dagger}\right) \ldots\left(B_{j_{2}}\right),
$$

and after formally taking the logarithm only terms of the form (4.1) can arise.

As a consequence we obtain the remarkably simple result

$$
\left\langle F_{n}\left|I_{m}\right| F_{n}\right\rangle=0
$$

\footnotetext{
${ }^{1} \mathrm{At}$ present there are no closed form results known for $I_{m}$ with arbitrary $m$. The cases $m=2$ and $m=3$ were computed in 39, the $m=2$ case is given in eq. 2.8). Therefore, at present explicit expressions can be written down only for $J_{1}^{\prime}, J_{2}^{\prime}$ and $J_{3}^{\prime}$. Higher charges and higher $J_{m}^{\prime}$ could be computed from the definition (2.7).
} 
which follows from ${ }_{j}\left\langle n\left|\left(B_{j}\right)^{k}\right| n\right\rangle_{j}=0$ for arbitrary $n, k$ and site $j$.

Applying (3.4) we find that in the quantum quench starting from the state $\left|F_{n}\right\rangle$ the resulting root density is constant and given simply by the total particle density:

$$
\rho_{r}(p)=n
$$

The hole density can be calculated from 2.15):

$$
\rho_{h}(p)=1,
$$

where we used $\int_{-\pi}^{\pi} \frac{d p}{2 \pi} \varphi(p)=1$. The filling fraction for these states is therefore

$$
\frac{\rho_{r}(p)}{\rho_{r}(p)+\rho_{h}(p)}=\frac{n}{1+n}
$$

A physical interpretation can be given as follows. In the initial states $\left|F_{n}\right\rangle$ all particles have well-defined positions. The initial states are not eigenstates, so the real-time dynamics of the system is non-trivial. Due to the initial sharp localization in real space we can expect that the eigenstates contributing to the dynamics will be spread out maximally in momentum space. Finding a completely constant root density in an interacting system is remarkable nevertheless, and it is a special property of the system and the initial states chosen.

It is very easy to give predictions for the long-time limit of the local operators $J_{m}^{\prime}$ defined in the previous section. If $\rho_{r}(p)$ is constant then the unique solution of (3.11) is $f(p)=0$, and from (3.13) we obtain

$$
\lim _{t \rightarrow \infty}\left\langle J_{m}^{\prime}(t)\right\rangle=\left\langle\rho_{G G E} J_{m}^{\prime}\right\rangle=0 .
$$

The operators $J_{m}^{\prime}$ are special in the sense that they also have zero mean value in the initial states:

$$
\left\langle J_{m}^{\prime}(0)\right\rangle=\left\langle\Psi_{0}\left|J_{m}^{\prime}\right| \Psi_{0}\right\rangle=0 .
$$

This follows from the fact that their structure is essentially the same as those of the charges. However, they are not conserved in time. For example it can be checked easily that

$$
\left.\frac{d}{d t}\left\langle J_{1}^{\prime}(t)\right\rangle\right|_{t=0}=i\left\langle\Psi_{0}\left|\left[H, J_{1}^{\prime}\right]\right| \Psi_{0}\right\rangle \neq 0 .
$$

Therefore the prediction that all $\left\langle J_{m}^{\prime}(t)\right\rangle$ approach zero in the long-time limit is a highly non-trivial statement, and it can be used as a test of the GGE.

For the sake of completeness we compute the Lagrange-multipliers for this GGE. All relevant functions are constant and from (3.7) we obtain

$$
\beta_{0}=\log \frac{1+n}{n}, \quad \text { and } \quad \beta_{m}=0 \text { for } \quad m \neq 0 .
$$

To conclude this subsection we remark that any small departure from the sharp localization of the one-site particle numbers changes the resulting root densities. For example if the initial state is given by

$$
\left|\Psi_{0}\right\rangle=\otimes_{j=1}^{L} \frac{|1\rangle_{j}+\alpha|0\rangle_{j}}{\sqrt{1+|\alpha|^{2}}}
$$

then the mean values of the two simplest charges are

$$
\left\langle\Psi_{0}\left|I_{1}\right| \Psi_{0}\right\rangle=\left\langle\Psi_{0}\left|I_{-1}\right| \Psi_{0}\right\rangle=\chi^{2} \frac{|\alpha|^{2}}{\left(1+|\alpha|^{2}\right)^{2}} .
$$

According to (3.4) the root density $\rho(p)$ will have a non-vanishing first Fourier component for any $\alpha \neq 0$. 


\subsection{Breaking translational invariance}

Let us define initial states which are not translationally invariant, but still have fixed one-site particle numbers. As examples we consider the states

$$
\left|F_{10}\right\rangle=\otimes_{j=1}^{L / 2}\left(|1\rangle_{2 j} \otimes|0\rangle_{2 j-1}\right) \quad \text { and } \quad\left|F_{20}\right\rangle=\otimes_{j=1}^{L / 2}\left(|2\rangle_{2 j} \otimes|0\rangle_{2 j-1}\right) .
$$

These states are invariant under translation by two sites. It is an important question whether the full translational invariance gets restored in the long-time limit. In Section 7 we consider the free boson theory $(q=1)$ and demonstrate on a few simple examples that mean values of local operators become translationally invariant indeed. In the cases $q>1$ most of the hopping amplitudes in the Hamiltonian are smaller than in the free case, but the particles can still hop from any site to its neighbours irrespective of the occupation number. Also, there is no one-site potential or any other term in the Hamiltonian which could "freeze" the artificial order of the initial state. Therefore we conjecture that translational invariance gets restored for any $q \geq 1$.

The GGE predictions for the steady state are derived easily. Mean values of the charges $I_{m}, m>1$ are all zero due to the same reasons as in the previous subsection. Therefore the resulting root densities are constant and only depend on the average particle number. For example $\rho(p)=1 / 2$ and $\rho(p)=1$ for $\left|F_{10}\right\rangle$ and $\left|F_{20}\right\rangle$, respectively.

It follows from our considerations that the predictions of the GGE are completely the same for any two initial states, if both are products of one-site particle number eigenstates and the overall particle density is the same. For example the initial states $\left|F_{1}\right\rangle$ and $\left|F_{20}\right\rangle$ should lead to the same long-time limit for any local quantity. This is a surprising statement, and it can be used as a check of the GGE.

\section{$5 \quad$ Large $q$ limit: Equilibrium properties}

In this section we treat the $q \rightarrow \infty$ limit of the model. We review the special properties of this limiting case, and also establish new results for a specific local operator: the Emptiness Formation Probability. These results serve as a basis to study quantum quenches in the large $q$ limit, which is considered in the next section.

In the $q \rightarrow \infty$ limit the local $q$-boson operators behave as

$$
B_{j} \rightarrow \phi_{j} \quad B_{j}^{\dagger} \rightarrow \phi_{j}^{\dagger},
$$

where the operators $\phi_{j}, \phi_{j}^{\dagger}$ are defined by their action

$$
\phi_{j}|n\rangle_{j}=|n-1\rangle_{j} \quad \phi_{j}^{\dagger}|n\rangle_{j}=|n+1\rangle_{j} .
$$

The Lax-operator is written as

$$
L(\lambda)=\left(\begin{array}{cc}
e^{\lambda} & \phi^{\dagger} \\
\phi & e^{-\lambda}
\end{array}\right)
$$

and the Hamiltonian is

$$
H=-\sum_{j=1}^{L}\left(\phi_{j}^{\dagger} \phi_{j+1}+\phi_{j+1}^{\dagger} \phi_{j}-2 N_{j}\right) .
$$

This model attracted considerable attention, partly because it is closely related to the combinatorial problem of plane partitions [49, 50, 51, 52]. In the literature it is often called the phase model.

The Algebraic Bethe Ansatz solution was first given in [53], where equilibrium correlation functions were computed as well. The coordinate space wave functions were first computed in [49], where it was shown that they are given by Schur polynomials. In our notations the coordinate Bethe Ansatz wave function can be written as

$$
\left|\{p\}_{N}\right\rangle=\prod_{j=1}^{N} B\left(\lambda_{j}\right)|0\rangle=\sum_{1 \leq x_{1} \leq x_{2} \leq \ldots x_{N} \leq L} C_{N}\left(x_{1}, \ldots, x_{N}\right) \phi_{x_{1}}^{\dagger} \ldots \phi_{x_{N}}^{\dagger}|0\rangle,
$$


where the coefficients are

$$
C_{N}\left(x_{1}, \ldots, x_{N}\right)=\frac{1}{\left(\prod_{j} e^{\lambda_{j}}\right)^{L+1}} \frac{\operatorname{det}_{N}\left(\left(a_{j}\right)^{k+x_{k}}\right)}{\operatorname{det}_{N}\left(\left(a_{j}\right)^{k}\right)},
$$

where

$$
a_{j}=e^{2 \lambda_{j}}=e^{i p_{j}} .
$$

The Bethe equations take the following simple form:

$$
\left(a_{j}\right)^{L+N}=(-1)^{N-1} \prod_{k=1}^{N} a_{k} .
$$

This can be obtained from the $q \rightarrow \infty$ limit of (2.10), or directly from the ABA developed for the phase model [53].

If the rapidities satisfy the Bethe equations, then the norm of the Bethe state (5.2) is

$$
\left\langle\{p\}_{N} \mid\{p\}_{N}\right\rangle=L(L+N)^{(N-1)} \prod_{i<j}\left|a_{i}-a_{j}\right|^{-2} .
$$

This result was obtained in [53] using the Algebraic Bethe Ansatz, whereas in [49] it was shown that it follows from certain properties of the Schur polynomials. The energy eigenvalues are given by

$$
E_{N}=\sum_{j=1}^{N} e\left(p_{j}\right) \quad \text { where } \quad e(p)=4 \sin ^{2}(p / 2),
$$

whereas the eigenvalues for the higher charges are

$$
\left\langle I_{m}\right\rangle=\frac{1}{|m|} \sum_{j=1}^{N} e^{-i m p_{j}} .
$$

We also note that in the thermodynamic limit the relation between the root and hole densities is simply

$$
\rho_{r}(p)+\rho_{h}(p)=1+n,
$$

which follows from the $q \rightarrow \infty$ limit of (2.15) or directly from the Bethe equations (5.4).

Results for correlation functions were also computed in $\mid 53]$ and [49, 50, 51]. Here we consider the $m$-site Emptiness Formation Probability (EFP), which is the probability to have zero occupancy number on $m$ neighbouring sites. We derive new formulas for the onesite and two-site EFP in arbitrary excited states. These results are used in the next section to give the GGE predictions in the quench problems.

Let us define the operators $\Pi_{j}$ which project to the zero-particle state on site $j$. Their action is simply

$$
\Pi_{j}|n\rangle_{j}=\delta_{n, 0}|0\rangle_{j}
$$

The m-site EFP operator is given by

$$
E^{(m)}=\prod_{j=1}^{m} \Pi_{j}
$$

In 53 it was shown that the normalized $m$-site EFP in a Bethe state is

$$
\left\langle\{p\}_{N}\left|E^{(m)}\right|\{p\}_{N}\right\rangle=(1+n) \operatorname{det} Y^{(m)}
$$

with

$$
Y_{j k}^{(m)}=\delta_{j, k}-\frac{1}{L} \frac{1}{1+n} \frac{\sin \left(\frac{m+1}{2}\left(p_{j}-p_{k}\right)\right)}{\sin \left(\frac{1}{2}\left(p_{j}-p_{k}\right)\right.}
$$

and $n=N / L$. The thermodynamic limit of this expression is a Fredholm determinant. 
Here we consider the two simplest cases $m=1$ and $m=2$ and show that the determinant can be expressed using single sums over the particles. In the thermodynamic limit we thus obtain the EFP's as sums of products of simple integrals. This is a huge simplification as opposed to the original result of a full Fredholm determinant. For simplicity we only consider states with zero total momentum, but this does not change the conclusions.

In the $m=1$ case we have

$$
Y_{j k}^{(1)}=\delta_{j, k}-\frac{1}{L} \frac{1}{1+n} 2 \cos \left(\frac{1}{2}\left(p_{j}-p_{k}\right)\right) .
$$

Multiplying the $j$ th row with $e^{i p_{j} / 2}$ and the $k$ th column with $e^{i p_{k} / 2}$ leads to

$$
\tilde{Y}_{j k}^{(1)}=\delta_{j, k} a_{j}-F_{j k}, \quad \text { with } \quad F_{j k}=\frac{1}{L} \frac{1}{1+n}\left(a_{j}+a_{k}\right),
$$

The matrix $F$ has rank 2, therefore in the expansion of the determinant we only have terms where at most 2 elements are chosen from $F$. This leads to

$$
\operatorname{det} \tilde{Y}_{j k}^{(1)}=1-\sum_{j=1}^{N} \frac{1}{L} \frac{1}{1+n} 2-\sum_{j<k} \frac{1}{a_{j} a_{k}}\left(\frac{1}{L} \frac{1}{1+n}\right)^{2}\left(a_{j}-a_{k}\right)^{2},
$$

where we used $\prod_{j} a_{j}=1$. For the finite volume EFP we thus obtain

$$
\left\langle\{p\}_{N}\left|E^{(1)}\right|\{p\}_{N}\right\rangle=\frac{1}{1+n}-\frac{1}{L^{2}} \frac{1}{1+n} \sum_{j, k} \frac{a_{j}}{a_{k}} .
$$

Let us define renormalized higher charges as

$$
Q_{m}=|m| \frac{\left\langle I_{m}\right\rangle}{L}=\frac{1}{L} \sum_{j=1}^{N} e^{-i m p_{j}},
$$

where $I_{m}$ is given by (5.6). In the thermodynamic limit we have

$$
Q_{m}=\int_{-\pi}^{\pi} \frac{d p}{2 \pi} \rho(p) e^{-i m p}
$$

Using this definition the one-site EFP is expressed simply as

$$
\left\langle E^{(1)}\right\rangle=\frac{1}{1+n}\left(1-\left|Q_{1}\right|^{2}\right) .
$$

Formula (5.11) is valid both in finite volume and in the thermodynamic limit. It is understood that (5.9) or (5.10) has to be used depending on the situation.

We now calculate the two-site EFP. As a first step we write the corresponding determinant as

$$
\operatorname{det} Y_{j k}^{(2)}=\prod_{j} a_{j}^{-2} \times \operatorname{det} \tilde{Y}_{j k}^{(2)},
$$

where now

$$
\tilde{Y}_{j k}^{(2)}=\delta_{j, k} a_{j}^{2}-F_{j k}, \quad \text { with } \quad F_{j k}=\frac{1}{L} \frac{1}{1+n}\left(a_{j}^{2}+a_{j} a_{k}+a_{k}^{2}\right),
$$

The matrix $F$ has at most rank 3 , because it is a sum of three matrices with rank 1 . Therefore in the expansion of the determinant it is enough keep terms where at most 3 elements are chosen from $F$. This leads to

$$
\begin{aligned}
\operatorname{det} Y_{j k}^{(2)}= & 1-\sum_{j=1}^{N} \frac{1}{L} \frac{1}{1+n} 3+\sum_{j<k} \frac{1}{L^{2}} \frac{1}{(1+n)^{2}}\left(6-\frac{a_{j}^{2}}{a_{k}^{2}}-\frac{a_{k}^{2}}{a_{j}^{2}}-2 \frac{a_{j}}{a_{k}}-2 \frac{a_{k}}{a_{j}}\right)- \\
& -\sum_{j<k<l} \frac{1}{L^{3}} \frac{1}{(1+n)^{3}}\left(\frac{a_{j}}{a_{k}}-\frac{a_{k}}{a_{j}}\right)^{2}\left(\frac{a_{j}}{a_{l}}-\frac{a_{l}}{a_{j}}\right)^{2}\left(\frac{a_{k}}{a_{l}}-\frac{a_{l}}{a_{k}}\right)^{2} .
\end{aligned}
$$


After some tedious but elementary calculations we obtain the EFP as

$$
\begin{aligned}
\left\langle\{p\}_{N}\left|E^{(2)}\right|\{p\}_{N}\right\rangle= & \frac{1}{(1+n)^{2}}-\frac{1}{L^{2}} \frac{2}{(1+n)^{2}} \sum_{j, k} \frac{a_{j}}{a_{k}}-\frac{1}{L^{2}} \frac{1}{(1+n)^{2}} \sum_{j, k} \frac{a_{j}^{2}}{a_{k}^{2}} \\
& -\frac{1}{L^{3}} \frac{1}{(1+n)^{2}} \sum_{j, k, l}\left(\frac{a_{j} a_{k}}{a_{l}^{2}}+\frac{a_{l}^{2}}{a_{j} a_{k}}\right) .
\end{aligned}
$$

Using the definition (5.9) this can be expressed as

$$
\left\langle\{p\}_{N}\left|E^{(2)}\right|\{p\}_{N}\right\rangle=\frac{1}{(1+n)^{2}}\left(1-2\left|Q_{1}\right|^{2}-\left|Q_{2}\right|^{2}-\left(\left(Q_{-1}\right)^{2} Q_{2}+\left(Q_{1}\right)^{2} Q_{-2}\right)\right) .
$$

This result remains valid in the thermodynamic limit if the definition (5.10) is used.

It is a special property of this system that the 1-site (or 2-site) EFP could be expressed using the mean values of the first (or first two) charges, respectively, and that the EFP does not depend on the other details of the states. We now argue that this is a general pattern: the $m$-site EFP only depends on the first $m$ charges and the overall particle density. Also we show how to obtain the results (5.11) and (5.12) directly in the thermodynamic limit.

In 53 it was shown that the thermodynamic limit of the formula (5.8) is the Fredholm determinant

$$
\left\langle E^{(m)}\right\rangle=(1+n) \operatorname{det}\left(1-\hat{T}^{(m)}\right)
$$

where $\hat{T}^{(m)}$ is an integral operator which acts on functions defined on $[-\pi, \pi]$ as

$$
\left(\hat{T}^{(m)} f\right)(p)=\int_{-\pi}^{\pi} \frac{d q}{2 \pi} T^{(m)}(p, q) f(q)
$$

where

$$
T^{(m)}(p, q)=\frac{1}{1+n} \rho(y) K^{(m)}(p, q) \quad \text { with } \quad K^{(m)}(p, q)=\frac{\sin \left(\frac{m+1}{2}(p-q)\right)}{\sin \left(\frac{1}{2}(p-q)\right)} .
$$

The Fredholm determinant can be expressed as

$$
\operatorname{det}\left(1-\hat{T}^{(m)}\right)=\sum_{k=0}^{\infty} \frac{(-1)^{k}}{k !} \frac{1}{(1+n)^{k}}\left(\prod_{j=1}^{k} \int_{-\pi}^{\pi} d p_{j} \rho\left(p_{j}\right)\right) \operatorname{det} \Lambda_{k}^{(m)},
$$

where $\Lambda_{k}^{(m)}$ is a $k$-by- $k$ matrix with elements given by

$$
\left(\Lambda_{k}^{(m)}\right)_{a b}=K^{(m)}\left(p_{a}, p_{b}\right)=\frac{e^{i \frac{m p_{a}}{2}}}{e^{i \frac{m p_{b}}{2}}}+\frac{e^{i \frac{(m-2) p_{a}}{2}}}{e^{i \frac{(m-2) p_{b}}{2}}}+\cdots+\frac{e^{i \frac{m p_{b}}{2}}}{e^{i \frac{m p_{a}}{2}}} .
$$

Note that $\Lambda_{k}^{(m)}$ is a sum of $m+1$ matrices with rank 1 , therefore its determinant is identically zero if $k>m+1$. It is thus enough to keep the terms $k \leq m+1$ in (5.13), which can be evaluated systematically using the formula (5.14). Note that for any $1 \leq a \leq k$ the variable $p_{a}$ only appears in the $a$ th row or the ath column of $\Lambda_{k}^{(m)}$. Therefore the highest power of $e^{i p_{a}}$ to appear in the determinant is $e^{i m p_{a}}$. It follows that the $m$-site EFP only depends on the overall particle number and the charges $Q_{l}$ with $|l| \leq m$.

\section{Large $q$ limit: Quantum Quenches}

In this section we investigate the quantum quenches in the $q \rightarrow \infty$ limit of the system. In two cases we prove rigorously that the GGE provides correct predictions for the stationary states. For the quench from $\left|\Psi_{0}\right\rangle=\left|F_{1}\right\rangle$ we also compute the exact time-dependence of the one-site EFP. 


\subsection{Quantum quench from $\left|\Psi_{0}\right\rangle=\left|F_{1}\right\rangle$}

We consider the quench starting from the initial state

$$
\left|\Psi_{0}\right\rangle=\left|F_{1}\right\rangle=\otimes_{j=1}^{L}|1\rangle_{j},
$$

and evaluate the predictions of the Diagonal Ensemble (3.1) in the thermodynamic limit. We assume for simplicity that $L$ is even.

The overlaps with the initial state are non-zero only if $N=L$ and are given simply by a particular component of the Bethe vector (5.2) with coordinates $x_{k}=k$. Therefore, the normalized and squared overlaps are

$$
\frac{\left|\left\langle F_{1} \mid\{p\}_{N}\right\rangle\right|^{2}}{\left\langle\{p\}_{N} \mid\{p\}_{N}\right\rangle}=\frac{1}{N(2 N)^{(N-1)}}\left|\operatorname{det}\left(\left(a_{j}\right)^{2 k}\right)\right|^{2}=\frac{1}{N(2 N)^{(N-1)}} \prod_{j<k}\left|a_{j}^{2}-a_{k}^{2}\right|^{2} .
$$

In deriving (6.2) we used that $\left|\prod_{j} a_{j}\right|=1$.

The initial state is translationally invariant, therefore only states with $\prod_{j} a_{j}=1$ can have a non-zero overlap. For these states the Bethe equations (5.4) are

$$
a_{j}^{2 L}=-1 .
$$

Solutions are given by

$$
a_{j}=e^{i \frac{\pi\left(2 I_{j}-1\right)}{2 L}}, \quad I_{j}=1,2, \ldots 2 L .
$$

The zero momentum Bethe states are thus given by the subsets

$$
\{a\}_{L} \subset\{\omega\}_{2 L}, \quad \omega_{k}=e^{i \frac{\pi(2 k-1)}{2 L}} \quad k=1,2, \ldots 2 L
$$

satisfying the constraint $\prod_{j} a_{j}=1$. The numbers $\omega_{k}$ can be paired such that

$$
\{\omega\}_{2 L}=\left\{\left(\omega_{k},-\omega_{k}\right)\right\}_{k=1 \ldots L} .
$$

It follows from formula (6.2) that the overlap is non-vanishing only if exactly one rapidity is chosen from each pair. In any other case at least one factor in (6.2) would be zero. Therefore, the states with non-vanishing overlap are given by

$$
a_{j}=s_{j} \omega_{j}, \quad \text { where } s_{j}= \pm 1, \quad j=1 \ldots L,
$$

with the constraint that the total momentum is zero. We have

$$
1=\prod_{j=1}^{N} a_{j}=\prod_{j=1}^{N}\left(s_{j} \omega_{j}\right)=e^{i \pi N / 2} \prod_{j=1}^{N} s_{j} .
$$

We assumed that $N$ is even, therefore the equation above can be satisfied by choosing the first $N-1$ signs arbitrarily and then fixing $s_{N}$ accordingly. It follows that there are a total number of $2^{N-1}$ states with non-vanishing overlap.

The overlaps are functions of the variables $a_{j}^{2}$, therefore they don't depend on the signs $s_{j}$. As a consequence, all non-vanishing overlaps are equal and we obtain

$$
\frac{\left|\left\langle F_{1} \mid\{p\}_{N}\right\rangle\right|^{2}}{\left\langle\{p\}_{N} \mid\{p\}_{N}\right\rangle}=\frac{1}{2^{N-1}}
$$

Comparing (6.5) to (5.2) we obtain the identity

$$
\prod_{1 \leq j<k \leq N}\left|e^{i \frac{2 j \pi}{N}}-e^{i \frac{2 k \pi}{N}}\right|^{2}=N^{N}
$$

As a check of our calculations we prove this identity directly. The l.h.s. can be written as

$$
\prod_{1<j \neq k \leq N}\left|e^{i \frac{2 j \pi}{N}}-e^{i \frac{2 k \pi}{N}}\right|=\left(\prod_{j=1}^{N-1}\left|1-e^{i \frac{2 j \pi}{N}}\right|\right)^{N} .
$$


Therefore we need to show that

$$
\prod_{j=1}^{N-1}\left|1-e^{i \frac{2 j \pi}{N}}\right|=N
$$

Consider the polynomial

$$
\prod_{j=1}^{N-1}\left(z-e^{i \frac{2 j \pi}{N}}\right)=\frac{\prod_{j=0}^{N-1}\left(z-e^{i \frac{2 j \pi}{N}}\right)}{z-1}=\frac{z^{N}-1}{z-1}=1+z+z^{2}+\cdots+z^{N-1} .
$$

In the second step we used that the product runs over all the roots of the polynomial $z^{N}-1$. Substituting $z=1$ into (6.7) completes the proof of (6.6).

With the solution (6.3) we have found the characterization of the states with nonvanishing overlaps. These overlaps are all equal, therefore all of these states have an equal weight in the Diagonal Ensemble. In the thermodynamic limit the ensemble will be dominated by those states where the signs $s_{j}$ are chosen randomly (without any particular pattern depending on the rapidity), and this leads to a constant root density in rapidity space:

$$
\rho_{r}(p)=1 .
$$

This coincides with the prediction of the GGE for this particular initial state. For the hole density we obtain $\rho_{h}(p)=1$ from (5.7), and the filling fraction is $1 / 2$. This is in agreement with the description of the states in terms of (6.3).

We stress that even though the GGE prediction for the root density has been confirmed, this does not mean that the Diagonal Ensemble is equal to the GGE. The GGE density matrix produces all states with $\rho_{r}(p)=1$, whereas the DE only includes those states which satisfy the constraint $a_{j}^{2} \neq a_{k}^{2}$ for $j \neq k$. However, mean values of local operators only depend on the root density, therefore the two ensembles lead to the same predictions.

The Emptiness Formation Probability is a physical observable with a non-trivial timedependence. In the inital state

$$
\left\langle\Psi_{0}\left|E^{(m)}\right| \Psi_{0}\right\rangle=0, \quad m=1,2, \ldots
$$

The GGE predictions for the long time limit can be calculated using the results of the previous section. For the two simplest cases we obtain from (5.11) and (5.12)

$$
\lim _{t \rightarrow \infty}\left\langle\Psi_{0}\left|E^{(1)}(t)\right| \Psi_{0}\right\rangle=\frac{1}{2} \quad \text { and } \quad \lim _{t \rightarrow \infty}\left\langle\Psi_{0}\left|E^{(2)}(t)\right| \Psi_{0}\right\rangle=\frac{1}{4} .
$$

In this particular quench problem it is possible to go further and compute the exact time dependence of these quantities. In the following we derive an exact result for $\left\langle\Psi_{0}\left|E^{(1)}(t)\right| \Psi_{0}\right\rangle$. The computation of 2-site EFP is left for further work.

\subsubsection{One-site EFP - Exact time evolution}

Form factors of $E_{m}$ were calculated in both [53] and [49]. The matrix element between two un-normalized off-shell Bethe states with arbitrary rapidities $\left|\left\{p^{B}\right\}_{N}\right\rangle$ and $\left|\left\{p^{C}\right\}_{N}\right\rangle$ reads

$$
\begin{aligned}
\left\langle\left\{p^{C}\right\}_{N}\left|E^{(m)}\right|\left\{p^{B}\right\}_{N}\right\rangle & =\left\langle 0\left|\left(\prod_{j=1}^{N} C\left(p_{j}^{C}\right)\right) E^{(m)}\left(\prod_{j=1}^{N} B\left(p_{j}^{B}\right)\right)\right| 0\right\rangle= \\
& =\frac{\prod_{j=1}^{L} e^{i\left(i p_{j}^{B}+p_{j}^{C}\right) / 2}}{\prod_{j<k}\left(e^{i p_{j}^{B}}-e^{i p_{k}^{B}}\right)\left(e^{i p_{k}^{C}}-e^{i p_{j}^{C}}\right)} \operatorname{det} T^{(m)}\left(\left\{p^{C}\right\}_{N},\left\{p^{B}\right\}_{N}\right),
\end{aligned}
$$

where

$T_{j k}^{(m)}\left(\left\{p^{C}\right\}_{N},\left\{p^{B}\right\}_{N}\right)=\frac{1}{e^{i p_{k}^{C}}-e^{i p_{j}^{B}}}\left(e^{i\left((2 N+2 L-1) p_{k}^{C}+p_{j}^{B}\right) / 2}-e^{i\left((2 N+2 L-2 m-1) p_{j}^{B}+(2 m+1) p_{k}^{C}\right) / 2}\right)$. 
If two rapidities coincide then the corresponding matrix element has to be evaluated using the l'Hôpital rule. For example if $p_{j}^{B} \rightarrow p_{k}^{C}$ :

$$
T_{j k}^{(m)} \rightarrow(N+L-m-1) e^{i(N+L-1) p_{k}^{C}} .
$$

If all rapidities coincide then we obtain the diagonal matrix elements, which after normalization and using the Bethe equations lead to (5.8).

In (6.8) the state on the l.h.s. is a dual vector, but it is not the complex conjugate of the ket vectors. In fact we have the norm formula for on-shell states

$$
\left\langle 0\left|\prod_{j=1}^{N} C\left(p_{j}\right) \prod_{j=1}^{N} B\left(p_{j}\right)\right| 0\right\rangle=e^{i P N} L(L+N)^{(N-1)} \prod_{j \neq k} \frac{1}{e^{i p_{k}}-e^{i p_{j}}},
$$

where $P=\sum_{j} p_{j}$.

If both states are on-shell then

$$
\operatorname{det} T^{(1)}\left(\left\{p^{C}\right\}_{N},\left\{p^{B}\right\}_{N}\right)=(N+L)^{N} \operatorname{det} \tilde{T}^{(1)}\left(\left\{p^{C}\right\}_{N},\left\{p^{B}\right\}_{N}\right)
$$

with

$$
\tilde{T}^{(1)}\left(\left\{p^{C}\right\}_{N},\left\{p^{B}\right\}_{N}\right)=F+G,
$$

where

$$
F_{j k}=-\frac{e^{i p_{k}^{C}}+e^{i p_{j}^{B}}}{N+L}
$$

and

$$
G_{j k}=\left\{\begin{array}{lll}
0 & \text { if } & p_{k}^{C} \neq p_{j}^{B} \\
e^{-i p_{k}^{C}} & \text { if } & p_{k}^{C}=p_{j}^{B}
\end{array}\right.
$$

We assumed here that $N$ is even. The matrix $F$ has rank 2, therefore the determinant is non-vanishing only if the rank of $G$ is at least $N-2$. This means that $G$ must have at least $N-2$ elements, therefore at least $N-2$ rapidities must coincide in the two states.

We consider the time evolution of the one-site EFP if the system is quenched from the initial state (6.1). We parametrize the rapidities of states with non-vanishing overlaps as

$$
p_{j}=c_{j}+u_{j} \pi, \quad \text { where } \quad c_{j}=\frac{\pi(2 j-1)}{2 L} \quad \text { and } \quad u_{j}=0,1 .
$$

The parameters $u_{j}$ can be chosen arbitrarily with the condition

$$
1=\prod_{j=1}^{N} e^{i p_{j}}=e^{i \pi N / 2} e^{i \pi \sum_{j=1}^{N} u_{j}}
$$

The spectral expansion in a finite volume reads

$\left\langle\Psi_{0}\left|E^{(1)}(t)\right| \Psi_{0}\right\rangle=\sum_{\left\{u^{B}\right\}_{N}} \sum_{\left\{u^{C}\right\}_{N}} \frac{e^{i t \sum_{j=1}^{N}\left(e\left(p_{j}^{B}\right)-e\left(p_{j}^{C}\right)\right)}}{2^{N-2}} D \prod_{j=1}^{N}\left(e^{-i p_{j}^{C} / 2} e^{-3 i p_{j}^{B} / 2}\right) \operatorname{det}_{N} \tilde{T}^{(1)}\left(\left\{p^{C}\right\}_{N},\left\{p^{B}\right\}_{N}\right)$,

where the parametrization (6.11) is implicit and $D$ is a sign factor depending on the ordering of the rapidities:

$$
D=\frac{\sqrt{\prod_{j \neq k}\left(e^{i p_{j}^{B}}-e^{i p_{k}^{B}}\right)\left(e^{i p_{k}^{C}}-e^{i p_{j}^{C}}\right)}}{\prod_{j<k}\left(e^{i p_{j}^{B}}-e^{i p_{k}^{B}}\right)\left(e^{i p_{k}^{C}}-e^{i p_{j}^{C}}\right)} .
$$

The determinant is non zero if there are at least $N-2$ coinciding rapidity pairs: $p_{j}^{B}=p_{k}^{C}$ with some $j, k$. Due to total momentum conservation there are only two possibilities: Either the two states are exactly the same, or there are two rapidities which differ. We treat these two cases separately. 
In the first case we have the diagonal elements

$$
\operatorname{det}_{N} \tilde{T}^{(1)}\left(\{p\}_{N},\{p\}_{N}\right)=-\frac{1}{4 N^{2}} \sum_{j<k} \frac{\left(e^{i p_{j}}-e^{i p_{k}}\right)^{2}}{e^{i p_{j}} e^{i p_{k}}}=\frac{1}{2 N^{2}} \sum_{j<k}\left(1-\cos \left(p_{j}-p_{k}\right)\right) .
$$

Here we used (6.4) again. The contribution of these terms in (6.13) is

$$
\sum_{\{u\}_{N}} \frac{1}{2^{N-1}} \frac{1}{N^{2}} \sum_{j<k}\left(1-\cos \left(p_{j}-p_{k}\right)\right) .
$$

Using the parametrization $(6.11)$ we have

$$
\sum_{\{u\}_{N}} \frac{1}{2^{N-1}} \frac{1}{N^{2}} \sum_{j<k}\left(1-\cos \left(c_{j}-c_{k}+\left(u_{j}-u_{k}\right) \pi\right)\right),
$$

where the sum runs over all sets of signs satisfying (6.12). The sums can be exchanged and we obtain

$$
\frac{1}{2^{N-1}} \frac{1}{N^{2}} \sum_{j<k} \sum_{\{u\}_{N}}\left(1-\cos \left(c_{j}-c_{k}+\left(u_{j}-u_{k}\right) \pi\right)\right)=\frac{1}{2^{N-1}} \frac{1}{N^{2}} \sum_{j<k} 2^{N-1}=\frac{N-1}{2 N} .
$$

We now consider the cases where there are two rapidity differences. Let $a$ and $b$ with $a<b$ denote the positions of the differences. Then the rapidities can be parametrized as

$$
p_{j}^{B}=\left\{\begin{array}{ll}
c_{j}+u_{j} \pi & \text { if } j \neq a, b \\
c_{a}+u_{a} \pi & \text { if } j=a \\
c_{b}+u_{b} \pi & \text { if } j=b
\end{array} \quad \text { and } \quad p_{j}^{C}=\left\{\begin{array}{ll}
c_{j}+u_{j} \pi & \text { if } j \neq a, b \\
c_{a}+u_{a} \pi+\pi & \text { if } j=a \\
c_{b}+u_{b} \pi+\pi & \text { if } j=b
\end{array},\right.\right.
$$

where condition $(6.12)$ is assumed. The determinant is

$$
\operatorname{det}_{N} \tilde{T}^{(1)}\left(\left\{p^{C}\right\}_{N},\left\{p^{B}\right\}_{N}\right)=-\frac{1}{2 N^{2}}\left(1-\cos \left(c_{a}-c_{b}+\left(u_{a}-u_{b}\right) \pi\right)\right) .
$$

The contribution of these cases to the EFP is

$$
-\frac{1}{N^{2}} \sum_{a<b} \sum_{\{u\}_{N}} \frac{1}{2^{N-1}} e^{-4 i t\left(\cos \left(c_{a}+u_{a} \pi\right)+\cos \left(c_{b}+u_{b} \pi\right)\right)}\left(1-\cos \left(c_{a}-c_{b}+\left(u_{a}-u_{b}\right) \pi\right)\right),
$$

where we used that

$$
e(p)-e(p+\pi)=-4 \cos (p) .
$$

The summation over the $u$ variables can be performed leading to

$$
-\frac{1}{N^{2}} \sum_{a<b}\left[\cos \left(4 \cos \left(c_{a}\right) t\right) \cos \left(4 \cos \left(c_{b}\right) t\right)+\sin \left(4 \cos \left(c_{a}\right) t\right) \sin \left(4 \cos \left(c_{b}\right) t\right) \cos \left(c_{a}-c_{b}\right)\right] .
$$

Finally we obtain the finite-volume EFP as

$\left\langle\Psi_{0}\left|E^{(1)}(t)\right| \Psi_{0}\right\rangle=\frac{N-1}{2 N}-\frac{1}{N^{2}} \sum_{a<b}\left[\cos \left(4 \cos \left(c_{a}\right) t\right) \cos \left(4 \cos \left(c_{b}\right) t\right)+\sin \left(4 \cos \left(c_{a}\right) t\right) \sin \left(4 \cos \left(c_{b}\right) t\right) \cos \left(c_{a}-c_{b}\right)\right]$.

Alternatively this can be written as

$$
\left\langle\Psi_{0}\left|E^{(1)}(t)\right| \Psi_{0}\right\rangle=\frac{1}{2}-\frac{1}{2}\left(\frac{1}{N} \sum_{a} \cos \left(4 \cos \left(c_{a}\right) t\right)\right)-\frac{1}{2}\left|\frac{1}{N} \sum_{a} \sin \left(4 \cos \left(c_{a}\right) t\right) e^{i c_{a}}\right|^{2} .
$$

We can take the thermodynamic limit with a fixed $t$ :

$$
\left\langle\Psi_{0}\left|E^{(1)}(t)\right| \Psi_{0}\right\rangle=\frac{1}{2}-\frac{1}{2}\left(\int_{0}^{\pi} \frac{d p}{\pi} \cos (4 \cos (p) t)\right)^{2}-\frac{1}{2}\left|\int_{0}^{\pi} \frac{d p}{\pi} \sin (4 \cos (p) t) e^{i p}\right|^{2} .
$$

For the initial value this formula gives $\left\langle\Psi_{0}\left|E^{(1)}\right| \Psi_{0}\right\rangle=0$, as expected. In the long-time limit the integrals become oscillatory and we have

$$
\lim _{t \rightarrow \infty}\left\langle\Psi_{0}\left|E^{(1)}(t)\right| \Psi_{0}\right\rangle=\frac{1}{2} .
$$

This agrees with the GGE prediction.

To our best knowledge formula (6.16) is the first closed form result result for the real time dynamics of a local observable in a genuinely interacting infinite volume system. 


\subsection{Quench from $\left|\Psi_{0}\right\rangle=\left|F_{10}\right\rangle$}

Here we consider the quantum starting from the state $\left|F_{10}\right\rangle$ defined in $(4.3)$. The initial state is not translationally invariant, but we argued in the previous section that translational invariance is restored in the long time limit. Therefore we expect that the Diagonal Ensemble applies for the local operators.

The state $\left|F_{10}\right\rangle$ is invariant with respect to translation by two sites, therefore the only states with non-vanishing overlap are those with total pseudo-momentum equal to 0 or $\pi$. The particle number is $N=L / 2$. The normalized overlaps are computed from (5.3) with $x_{k}=2 k$ and the norm formula (5.5):

$$
\frac{\left|\left\langle F_{10} \mid\{p\}_{N}\right\rangle\right|^{2}}{\left\langle\{p\}_{N} \mid\{p\}_{N}\right\rangle}=\frac{1}{L(L+N)^{(N-1)}}\left|\operatorname{det}\left(\left(a_{j}\right)^{3 k}\right)\right|^{2}=\frac{1}{2 N(3 N)^{(N-1)}} \prod_{j<k}\left|a_{j}^{3}-a_{k}^{3}\right|^{2} .
$$

The Bethe equations take the form

$$
a_{j}^{3 N}=(-1)^{N-1} \prod_{k=1}^{N} a_{k} .
$$

For simplicity we assume that $N$ is even and first consider states with zero total momentum. In this case the solutions are of the form

$$
a_{j}=e^{\frac{i \pi\left(2 I_{j}-1\right)}{3 N}}, \quad I_{j}=1 \ldots 3 N .
$$

Therefore, the Bethe state is described by a subset

$$
\{a\}_{N} \subset\{w\}_{3 N}, \quad w_{j}=e^{\frac{i \pi(2 j-1)}{3 N}} .
$$

The numbers $w_{j}$ can be arranged in triplets as

$$
\{w\}_{3 N}=\left\{w_{j}, w_{j} e^{i \pi / 3}, w_{j} e^{2 i \pi / 3}\right\}_{j=1}^{N} .
$$

It follows from (6.18) that the overlap is non-vanishing whenever exactly one member is chosen from each triplet. There are a total number of $3^{N-1}$ such states. The overlap does not depend on these choices, and applying the identity (6.5) in this case leads to

$$
\frac{\left|\left\langle F_{10} \mid\{p\}_{N}\right\rangle\right|^{2}}{\left\langle\{p\}_{N} \mid\{p\}_{N}\right\rangle}=\frac{1}{2 \cdot 3^{N-1}} .
$$

It is easy to see that the same result is obtained for states with total pseudo-momentum equal to $\pi$. Therefore all states with non-vanishing overlap have an equal weight in the Diagonal Ensemble. In the infinite volume limit the DE will be dominated by states where the choices from the triplets are random, and this leads to

$$
\rho_{r}(p)=\frac{1}{2}, \quad \rho_{h}(p)=1, \quad \frac{\rho_{r}(p)}{\rho_{r}(p)+\rho_{h}(p)}=1 / 3 .
$$

This result agrees with the predictions of the GGE.

We also consider the long-time limit of the one-site and two-site EFP's. In the initial state the one-site EFP is not translationally invariant: it is equal to zero (one) on the even (odd) sites, respectively. We conjectured that translational invariance is restored in the long time limit, and from (5.11) we obtain

$$
\lim _{t \rightarrow \infty}\left\langle\Psi_{0}\left|E^{(1)}(t)\right| \Psi_{0}\right\rangle=\frac{2}{3} .
$$

On the other hand, the two-site EFP is translationally invariant at all times, its initial value is zero, and for the long time limit we obtain from (5.12)

$$
\lim _{t \rightarrow \infty}\left\langle\Psi_{0}\left|E^{(2)}(t)\right| \Psi_{0}\right\rangle=\frac{4}{9} .
$$

The calculation of the exact time dependence is more challenging in this case, because the matrix elements between states with different total momentum have a more complicated structure than those treated in 6.1.1. We leave this problem to further research. 


\section{$7 \quad$ Free bosons}

Here we consider the model in the $q \rightarrow 1$ limit, which is a free bosonic theory defined by the Hamiltonian

$$
H=-\sum_{j=0}^{L-1}\left(b_{j}^{\dagger} b_{j+1}+b_{j+1}^{\dagger} b_{j}-2 N_{j}\right) .
$$

For later convenience we indexed the sites in 7.1 from 0 to $(L-1)$.

It was shown in [16] that if a model is quenched from any state to a free Hamiltonian, then the GGE holds whenever the initial state satisfies the cluster decomposition principle. The proof of [16] also applies to the lattice model of free bosons, and the initial states considered in the previous sections satisfy the cluster decomposition principle. Therefore the GGE must be valid in these cases.

The goal of the present section is to derive explicit formulas for the time dependence of simple observables, and to demonstrate that the GGE predictions are indeed correct, and that translational invariance is restored in the large time limit. We consider two initial states:

$$
\left|\Psi_{0}\right\rangle=\left|F_{1}\right\rangle \quad \text { and } \quad\left|\Psi_{0}\right\rangle=\left|F_{20}\right\rangle .
$$

They have the same particle density $n=1$ and according to the GGE they should lead to the same stationary state. As physical observables we choose the one-site particle number operators $N_{j}$ and their square $N_{j}^{2}$. We first evaluate their exact time evolution, and then show that in the long time limit they approach the GGE predictions. The calculations below are straightforward and elementary. Nevertheless, we felt that it is useful to present them, so that both the $q \rightarrow \infty$ and $q=1$ points can be benchmarks for the generic $q$ case.

The model is diagonalized with the Fourier modes of the one-site bosonic operators:

$$
\tilde{b}_{k}=\frac{1}{\sqrt{L}} \sum_{n=0}^{L-1} b_{n} e^{-i 2 \pi k n / L} \quad \tilde{b}_{k}^{\dagger}=\frac{1}{\sqrt{L}} \sum_{n=0}^{L-1} b_{n}^{\dagger} e^{-i 2 \pi k n / L} .
$$

The following commutation relation holds:

$$
\left[\tilde{b}_{j}, \tilde{b}_{k}^{\dagger}\right]=\delta_{j, k} .
$$

In terms of the Fourier modes the Hamiltonian can be written as

$$
H=\sum_{k=0}^{L-1} \epsilon_{k} \tilde{b}_{k}^{\dagger} \tilde{b}_{k}
$$

where

$$
\epsilon_{k}=4 \sin ^{2}\left(p_{k} / 2\right), \quad \text { with } \quad p_{k}=\frac{2 \pi k}{L} .
$$

Therefore the time dependence of the operators (in the Heisenberg picture) is

$$
\tilde{b}_{k}^{\dagger}(t)=\tilde{b}_{k}^{\dagger} e^{-i \epsilon_{k} t} \quad \tilde{b}_{k}(t)=\tilde{b}_{k} e^{i \epsilon_{k} t} .
$$

We will consider the particle number operators on site 0 and 1 :

$$
N_{0}=\frac{1}{L} \sum_{j, k} \tilde{b}_{j}^{\dagger} \tilde{b}_{k} \quad N_{1}=\frac{1}{L} \sum_{j, k} \tilde{b}_{j}^{\dagger} \tilde{b}_{k} e^{2 \pi i(j-k) / L} .
$$

Their time-dependence is

$$
N_{0}(t)=\frac{1}{L} \sum_{j, k} \tilde{b}_{j}^{\dagger} \tilde{b}_{k} e^{i\left(\epsilon_{j}-\epsilon_{k}\right) t} \quad N_{1}(t)=\frac{1}{L} \sum_{j, k} \tilde{b}_{j}^{\dagger} \tilde{b}_{k} e^{2 \pi i(j-k) / L} e^{i\left(\epsilon_{j}-\epsilon_{k}\right) t} .
$$

For their squares we obtain

$$
\begin{aligned}
& N_{0}^{2}=\frac{1}{L^{2}} \sum_{j_{1}, j_{2}, k_{1}, k_{2}} \tilde{b}_{j_{1}}^{\dagger} \tilde{b}_{j_{2}}^{\dagger} \tilde{b}_{k_{1}} \tilde{b}_{k_{2}}+N_{0} \\
& N_{1}^{2}=\frac{1}{L^{2}} \sum_{j_{1}, j_{2}, k_{1}, k_{2}} \tilde{b}_{j_{1}}^{\dagger} \tilde{b}_{j_{2}}^{\dagger} \tilde{b}_{k_{1}} \tilde{b}_{k_{2}} e^{2 \pi i\left(j_{1}+j_{2}-k_{1}-k_{2}\right) / L}+N_{1},
\end{aligned}
$$


with the time dependence given by

$$
\begin{aligned}
& N_{0}^{2}(t)=\frac{1}{L^{2}} \sum_{j_{1}, j_{2}, k_{1}, k_{2}} \tilde{b}_{j_{1}}^{\dagger} \tilde{b}_{j_{2}}^{\dagger} \tilde{b}_{k_{1}} \tilde{b}_{k_{2}} e^{i\left(\epsilon_{j_{1}}+\epsilon_{j_{2}}-\epsilon_{k_{1}}-\epsilon_{k_{2}}\right) t}+N_{0}(t) \\
& N_{1}^{2}(t)=\frac{1}{L^{2}} \sum_{j_{1}, j_{2}, k_{1}, k_{2}} \tilde{b}_{j_{1}}^{\dagger} \tilde{b}_{j_{2}}^{\dagger} \tilde{b}_{k_{1}} \tilde{b}_{k_{2}} e^{2 \pi i\left(j_{1}+j_{2}-k_{1}-k_{2}\right) / L} e^{i\left(\epsilon_{j_{1}}+\epsilon_{j_{2}}-\epsilon_{k_{1}}-\epsilon_{k_{2}}\right) t}+N_{1}(t) .
\end{aligned}
$$

\subsection{Quench from $\left|\Psi_{0}\right\rangle=\left|F_{1}\right\rangle$}

In this case the initial state is given by

$$
\left|\Psi_{0}\right\rangle=\left|F_{1}\right\rangle=b_{l-1}^{\dagger} \ldots b_{1}^{\dagger} b_{0}^{\dagger}|0\rangle .
$$

This state is translationally invariant, therefore it is enough to consider the operators on site

0 . In calculating the local observables below we will make use of the commutation relations

$$
\left[\tilde{b}_{k}, b_{n}^{\dagger}\right]=\frac{1}{L} e^{-i 2 \pi k n / L}, \quad\left[b_{n}, \tilde{b}_{k}^{\dagger}\right]=\frac{1}{L} e^{i 2 \pi k n / L} .
$$

For the time dependence of the particle number operator we obtain

$$
\left\langle F_{1}\left|N_{0}(t)\right| F_{1}\right\rangle=\frac{1}{L^{2}} \sum_{j, k} e^{i\left(\epsilon_{j}-\epsilon_{k}\right) t} \sum_{n} e^{-i 2 \pi(k-j) n / L}=\frac{1}{L} \sum_{j} 1=1 .
$$

This is the expected result, because the total particle number is conserved and the system is translationally invariant at all times.

For the expectation value of $N_{0}^{2}$ we obtain

$$
\begin{aligned}
\left\langle F_{1}\left|N_{0}^{2}(t)\right| F_{1}\right\rangle & =\left\langle F_{1}\left|N_{0}(t)\right| F_{1}\right\rangle+\frac{1}{L^{4}} \sum_{j_{1}, j_{2}, k_{1}, k_{2}} e^{i\left(\epsilon_{j_{1}}+\epsilon_{j_{2}}-\epsilon_{k_{1}}-\epsilon_{k_{2}}\right) t} \sum_{n_{1}>n_{2}}\left(e^{-i 2 \pi\left(\left(k_{1}-j_{1}\right) n_{1}+\left(k_{2}-j_{2}\right) n_{2}\right) / l}+\text { perm. }\right) \\
& =3-\frac{2}{L^{3}} \sum_{j_{1}, j_{2}, k_{1}} e^{i\left(\epsilon_{j_{1}}+\epsilon_{j_{2}}-\epsilon_{k_{1}}-\epsilon_{j_{1}+j_{2}-k_{2}}\right) t} .
\end{aligned}
$$

Performing the infinite volume limit for fixed $t$ leads to

$$
\left\langle F_{1}\left|N_{0}^{2}(t)\right| F_{1}\right\rangle=3-2 \int_{-\pi}^{\pi} \frac{d p_{1}}{2 \pi} \frac{d p_{2}}{2 \pi} \frac{d q_{1}}{2 \pi} e^{i\left(\epsilon\left(p_{1}\right)+\epsilon\left(p_{2}\right)-\epsilon\left(q_{1}\right)-\epsilon\left(p_{1}+p_{2}-q_{1}\right)\right) t},
$$

where

$$
\epsilon(p)=4 \sin ^{2}(p / 2) .
$$

For $t=0$ the above formula yields $\left\langle F_{1}\left|N_{0}^{2}\right| F_{1}\right\rangle=1$, as expected. For $t \rightarrow \infty$ the integrals become strongly oscillatory and this leads to

$$
\lim _{t \rightarrow \infty}\left\langle F_{1}\left|N_{0}^{2}(t)\right| F_{1}\right\rangle=3 .
$$

\subsection{Quench from $\left|\Psi_{0}\right\rangle=\left|F_{20}\right\rangle$}

Here we consider the quench from the initial state

$$
\left|\Psi_{0}\right\rangle=\left|F_{20}\right\rangle=\frac{1}{\sqrt{2^{L / 2}}} b_{l-2}^{\dagger} b_{l-2}^{\dagger} \ldots b_{2}^{\dagger} b_{2}^{\dagger} b_{0}^{\dagger} b_{0}^{\dagger}|0\rangle .
$$

We compute the time evolution of the one-site particle number operators. The initial state is two-site shift invariant, therefore it is sufficient to consider the operators $N_{0}(t)$ and $N_{1}(t)$.

For the time evolution of $N_{0}(t)$ we obtain

$$
\begin{aligned}
\left\langle F_{20}\left|N_{0}(t)\right| F_{20}\right\rangle & =\frac{1}{2 L^{2}} \sum_{j, k} e^{i\left(\epsilon_{j}-\epsilon_{k}\right) t} \sum_{n=0}^{(l-2) / 2} 4 e^{-i 2 \pi(k-j) 2 n / L}= \\
& =\frac{1}{L} \sum_{j, k} e^{i\left(\epsilon_{j}-\epsilon_{k}\right) t}\left(\delta_{j, k}+\delta_{j-k, L / 2}\right)= \\
& =1+\frac{1}{L} \sum_{j} e^{i\left(\epsilon_{j}-\epsilon_{j+L / 2}\right) t} .
\end{aligned}
$$


In the thermodynamic limit this leads to

$$
\left\langle F_{20}\left|N_{0}(t)\right| F_{20}\right\rangle=1+\int_{-\pi}^{\pi} \frac{d p}{2 \pi} e^{i(\epsilon(p)-\epsilon(p+\pi)) t} .
$$

Similarly

$$
\begin{aligned}
\left\langle F_{20}\left|N_{1}(t)\right| F_{20}\right\rangle & =\frac{1}{2 L^{2}} \sum_{j, k} e^{i\left(\epsilon_{j}-\epsilon_{k}\right) t} e^{2 \pi i(j-k) / L} \sum_{n=0}^{(l-2) / 2} 4 e^{-i 2 \pi(k-j) 2 n / L}= \\
& =\frac{1}{L} \sum_{j, k} e^{i\left(\epsilon_{j}-\epsilon_{k}\right) t}\left(\delta_{j, k}-\delta_{j-k, L / 2}\right)= \\
& =1-\frac{1}{L} \sum_{j} e^{i\left(\epsilon_{j}-\epsilon_{j+L / 2}\right) t},
\end{aligned}
$$

leading to

$$
\left\langle F_{20}\left|N_{1}(t)\right| F_{20}\right\rangle=1-\int_{-\pi}^{\pi} \frac{d p}{2 \pi} e^{i(\epsilon(p)-\epsilon(p+\pi)) t} .
$$

In the initial state $\left\langle F_{20}\left|N_{0}\right| F_{20}\right\rangle=2$ and $\left\langle F_{20}\left|N_{1}\right| F_{20}\right\rangle=0$, whereas in the long time limit we have

$$
\lim _{t \rightarrow \infty}\left\langle F_{20}\left|N_{0}(t)\right| F_{20}\right\rangle=\lim _{t \rightarrow \infty}\left\langle F_{20}\left|N_{1}(t)\right| F_{20}\right\rangle=1 .
$$

For the squared operators similar but somewhat lengthier calculations result in

$$
\begin{aligned}
\left\langle F_{20}\left|N_{0}^{2}(t)\right| F_{20}\right\rangle= & 3+5 \int_{-\pi}^{\pi} \frac{d p}{2 \pi} e^{i(\epsilon(p)-\epsilon(p+\pi)) t}+2\left(\int_{-\pi}^{\pi} \frac{d p}{2 \pi} e^{i(\epsilon(p)-\epsilon(p+\pi)) t}\right)^{2}+ \\
& -3 \int_{-\pi}^{\pi} \frac{d p_{1}}{2 \pi} \frac{d p_{2}}{2 \pi} \frac{d q_{1}}{2 \pi} e^{i\left(\epsilon\left(p_{1}\right)+\epsilon\left(p_{2}\right)-\epsilon\left(q_{1}\right)-\epsilon\left(p_{1}+p_{2}-q_{1}\right)\right) t} \\
& -3 \int_{-\pi}^{\pi} \frac{d p_{1}}{2 \pi} \frac{d p_{2}}{2 \pi} \frac{d q_{1}}{2 \pi} e^{i\left(\epsilon\left(p_{1}\right)+\epsilon\left(p_{2}\right)-\epsilon\left(q_{1}\right)-\epsilon\left(p_{1}+p_{2}-q_{1}+\pi\right)\right) t}
\end{aligned}
$$

and

$$
\begin{aligned}
\left\langle F_{20}\left|N_{1}^{2}(t)\right| F_{20}\right\rangle= & 3-5 \int_{-\pi}^{\pi} \frac{d p}{2 \pi} e^{i(\epsilon(p)-\epsilon(p+\pi)) t}+2\left(\int_{-\pi}^{\pi} \frac{d p}{2 \pi} e^{i(\epsilon(p)-\epsilon(p+\pi)) t}\right)^{2}+ \\
& -3 \int_{-\pi}^{\pi} \frac{d p_{1}}{2 \pi} \frac{d p_{2}}{2 \pi} \frac{d q_{1}}{2 \pi} e^{i\left(\epsilon\left(p_{1}\right)+\epsilon\left(p_{2}\right)-\epsilon\left(q_{1}\right)-\epsilon\left(p_{1}+p_{2}-q_{1}\right)\right) t} \\
& +3 \int_{-\pi}^{\pi} \frac{d p_{1}}{2 \pi} \frac{d p_{2}}{2 \pi} \frac{d q_{1}}{2 \pi} e^{i\left(\epsilon\left(p_{1}\right)+\epsilon\left(p_{2}\right)-\epsilon\left(q_{1}\right)-\epsilon\left(p_{1}+p_{2}-q_{1}+\pi\right)\right) t}
\end{aligned}
$$

At $t=0$ we have $\left\langle F_{20}\left|N_{0}^{2}\right| F_{20}\right\rangle=4$ and $\left\langle F_{20}\left|N_{1}^{2}\right| F_{20}\right\rangle=0$, whereas in the long time limit

$$
\lim _{t \rightarrow \infty}\left\langle F_{20}\left|N_{0}^{2}(t)\right| F_{20}\right\rangle=\lim _{t \rightarrow \infty}\left\langle F_{20}\left|N_{1}^{2}(t)\right| F_{20}\right\rangle=3 .
$$

Thus we have demonstrated on these simple examples that non-trivial observables indeed become translationally invariant, and approach the same values as in the case of the initial state $\left|F_{1}\right\rangle$.

\subsection{GGE predictions}

In a free theory the GGE can be built conveniently using the mode occupation numbers. We define

$$
\rho_{G G E}=\frac{e^{-\sum_{j=0}^{L} \beta_{j} \tilde{I}_{j}}}{\operatorname{Tr}\left(e^{-\sum_{j=0}^{L} \beta_{j} \tilde{I}_{j}}\right)}, \quad \tilde{I}_{j}=\tilde{b}_{j}^{\dagger} \tilde{b}_{j} .
$$

This construction is equivalent to a GGE built from the local charges, which are the Fourier components of $\tilde{I}_{j}$ :

$$
I_{l}=\sum_{j=0}^{L-1} e^{i \frac{2 \pi j l}{L}} \tilde{I}_{j}=\sum_{n=0}^{L-1} b_{n+l}^{\dagger} b_{n}
$$


The Lagrange multipliers have to be fixed by the initial values of the charges, which are

$$
\left\langle F_{1}\left|\tilde{I}_{j}\right| F_{1}\right\rangle=\left\langle F_{20}\left|\tilde{I}_{j}\right| F_{20}\right\rangle=1 .
$$

On the other hand

$$
\operatorname{Tr}\left(\rho_{G G E} \tilde{I}_{j}\right)=\frac{\operatorname{Tr}\left(e^{-\beta_{j} \tilde{I}_{j}} \tilde{I}_{j}\right)}{\operatorname{Tr}\left(e^{-\beta_{j} \tilde{I}_{j}}\right)}=\frac{1}{e^{\beta_{j}}-1},
$$

which leads to $\beta_{j}=\log (2)$. All Lagrange multipliers are equal, therefore the GGE density matrix only depends on the total particle number operator. Note that the same result was found also in the interacting case, see eq. (4.2).

The GGE predictions for the operator $N_{0}^{2}$ can be evaluated using (7.2) and Wick's theorem and we find

$$
\operatorname{Tr}\left(\rho_{G G E} N_{0}^{2}\right)=3 .
$$

This result agrees with the asymptotic values derived in the previous two subsections, as expected.

\section{Discussion and Outlook}

In this paper we considered quantum quenches in the $q$-boson model. First we showed that the Generalized Eigenstate Thermalization Hypothesis holds in this system, therefore the GGE gives the correct asymptotic states if the initial state satisfies the cluster decomposition principle. The role of the latter is to ensure that the Diagonal Ensemble only includes states having the same mean values for the charges as the initial state.

Concentrating on simple initial states which have fixed one-site occupation numbers we were able to provide the GGE predictions. Surprisingly we found that for these initial states the resulting root densities are $\rho_{r}(p)=n$, where $n$ is the overall particle density. This result means that any two states within this family which have the same particle density will also have the same stationary behaviour.

We also considered the $q \rightarrow \infty$ limit of the system, where the exact overlaps are given by Schur polynomials, which can be expressed as determinants. For two initial states we were able to determine which states populate the Diagonal Ensemble. As a consequence we proved that in the thermodynamic limit the GGE predictions are correct. We believe that this is the first time that the GGE was proven to be valid in a model which is neither a free theory, nor solvable by free fermions. For the quench starting from $\left|\Psi_{0}\right\rangle=\left|F_{1}\right\rangle$ we calculated the exact time dependence of the one-site Emptiness Formation Probability (EFP). To our best knowledge this is the first time that an analytic result has been obtained for the real time dynamics of an observable in a genuinely interacting system, valid both in finite volume and in the thermodynamic limit.

A few comments about our results are in order.

The initial states that we considered are very special. They are not entangled at all, and they are pure Fock states in the local bosonic basis. However, they are very reasonable choices from a physical point of view. The states $\left|F_{n}\right\rangle$ are exact ground states of the infinitely repulsing Bose-Hubbard model with a given filling $n$, or they are approximate ground states for large repulsion parameters. Also, they could be realized in experimental situations. The state $\left|F_{10}\right\rangle$ can be considered as an analogue of the Néel state in the spin- $1 / 2 \mathrm{XXZ}$ spin chain, which has been the subject of a large body of recent theoretical works (see [19, 17, 54 and references therein). The XXZ model can be described as a theory of interacting fermions with attractive coupling, whereas here we dealt with an interacting bosonic system with repulsive coupling, so the physical behaviour can be markedly different. However, our initial states themselves are not more exceptional than the Néel state or other states considered elsewhere in the literature.

The $q$-boson model has been used previously as a lattice regularization of the continuum Lieb-Liniger model [39, 29]. Here we focused on the lattice model in its own right. In fact, all initial states become ill-defined if we take the scaling limit towards the Lieb-Liniger model, because they have fixed particle density in lattice units, which leads to infinite density in the 
continuum limit. One could consider a special limit where the initial state is also changed as we approach the Lieb-Liniger model, such that the final particle density is finite. This way we would obtain an initial state where particles are localized with Dirac-delta functions at a fixed distance from each other. However, these states are not normalizable and they excite Bethe states with arbitrarily high energy, therefore this would not be a well-defined quench problem either. We wish to stress that our initial states have just the opposite structure as those considered previously in [29, 31]: they are sharply localised in real space, as opposed to the Bose-Einstein condensate (BEC) states which are localised in momentum space.

In Section 6 we considered the $q \rightarrow \infty$ limit and in two cases we obtained the exact overlaps in a product form (eqs. (6.2) and (6.18)). The simplicity of this result is certainly a special property of both the model and the initial states, but already these cases show different behaviour than expected. In quantum quenches of the XXZ spin chain and the Lieb-Liniger model it was found that in the on-shell case (when the rapidities satisfy the Bethe equations) only those states have non-zero overlap which are formed out of $(p,-p)$ rapidity pairs [31, 55, 56]. In the $q$-boson model, on the other hand, we found completely different conditions. For example for $\left|\Psi_{0}\right\rangle=\left|F_{1}\right\rangle$ the requirement for the overlap to be non-vanishing is

$$
e^{2 i p_{j}} \neq e^{2 i p_{k}} \quad \text { for } \quad j \neq k .
$$

The $q \rightarrow \infty$ limit of the model is regular, the finite volume overlaps are continuous functions of $q$, therefore the pairing requirement does not hold for finite $q$ either.

In the previously considered quench problems the Quench Action method [18] was used to find the stationary states [31, 17, 19|. The Quench Action is a functional of the root and hole densities and is given by a combination of the overlaps and the micro-canonical entropy associated to a given root configuration. Finding the minimum of the QA provides the states which populate the system after the quench. In the XXZ chain and the Lieb-Liniger model the on-shell overlaps could be written in the form [31, 55, 56]

$$
\frac{\left|\left\langle\Psi_{0} \mid\{p\}_{N}\right\rangle\right|^{2}}{\left\langle\{p\}_{N} \mid\{p\}_{N}\right\rangle}=C\left(\{p\}_{N}\right) \prod_{j=1}^{N} v\left(p_{j}\right),
$$

where the pre-factor $C$ behaves as $\mathcal{O}\left(L^{0}\right)$ in the thermodynamic limit. This made it possible to write the QA as simple integrals over the root and hole densities, thus making a TBA-like analysis possible. In the present case the on-shell overlap formulas (6.5) and (6.19) are so simple that the conclusions could be drawn immediately and there was no need to apply the machinery of the QA approach. However, the non-zero on-shell overlaps are actually of the form (3.1). For example for $\left|\Psi_{0}\right\rangle=\left|F_{1}\right\rangle$ we have

$$
v(p)=\frac{1}{2} \quad \text { and } \quad C=2
$$

This trivially leads to a constant root density.

As a final comment we note that even though we confirmed the GGE hypothesis in the $q$-boson model, we did not make use of the GGE density matrix in any way. We assumed that the Diagonal Ensemble is valid, and argued that the GETH holds. Then the predictions for the long-time limit of the observables could be calculated as soon as the root density $\rho_{r}(p)$ was obtained from the charges. The generalized TBA equations (3.5) were only used to calculate the Lagrange multipliers. Therefore it might be more appropriate to call our results the "DE + GETH predictions" instead of the "predictions of the GGE". This behaviour might be a generic feature of interacting integrable models: If the GETH holds, then there must be a one-to-one correspondence between the charges and the root densities, therefore the GGE density matrix and the associated TBA equations are not needed. On the other hand, if the GETH does not hold, then the TBA analysis of the GGE density matrix is expected to give wrong predictions [23, 24].

Below we list a number of open questions, which deserve further investigation.

- For a generic $q$ is there a formula of the form (8.1) for the overlaps? The wave functions are given by Hall-Littlewood functions [40], and the overlaps with the pure Fock states 
are specific components of the wave function. It might be possible that determinant formulas could be found, if one makes use of the Bethe equations. Note that the Quench Action method can only agree with the GGE if the function $v(p)$ appearing in (3.1) is a constant. In any other case the resulting root density would not be constant, and this would contradict the GGE prediction.

- Results for local correlation functions of the $q$-boson model are very limited. Mean values are only known for the charges and their derivatives with respect to $\eta=\log (q)$ (see Subsection 3.1). It would be useful to derive new results for other operators, for example the $m$-site Emptiness Formation Probability.

- If the initial state is not translationally invariant, does this symmetry get restored in the long time limit? We argued that it does, but were not able to give a proof.

- Is it possible to derive GGE predictions for other initial states? There are no closed form formulas for the higher charges, but a truncated GGE can be established with the already available results [29. In the case of the XXZ spin chain a generating function for the higher charges was constructed in 21] and it was shown how to compute it for simple product states. It is an open question whether the method of [21] can be generalized to the $q$-boson model.

- In the $q \rightarrow \infty$ limit both the overlaps and the matrix elements of local operators are known and they take a relatively simple form $[53,49$, 51]. Moreover, the Bethe equations can be solved explicitly. These two properties allowed us to compute the time-dependence of the one-site EFP in the case of $\left|\Psi_{0}\right\rangle=\left|F_{1}\right\rangle$. We believe that this result could be extended to other operators. For example, the $m$-site EFP could be calculated with the same methods, such that result could be expressed as sums of products of simple integrals. It is an open question, whether exact calculations are possible for other operators or other initial states.

- In Section 5 we found that in the $q \rightarrow \infty$ limit the excited state mean values of the $m$-site EFP only depend on the mean values of the first $m$ charges and the particle density. This opens up a way to give exact GGE predictions even for those initial states where the full root density can not be reconstructed. For example, if the mean value of the first charge $I_{1}=\sum_{j} \phi_{j}^{\dagger} \phi_{j+1}$ can be calculated in the initial state, then (5.11) already gives the exact prediction for the long-time limit of the 1-site EFP. This could be used as a further check of the Diagonal Ensemble and the GGE, if the time evolution could be simulated by independent numerical methods.

Also, the relation between the EFP and the charges deserves further attention. It would be interesting to find a closed form result for the $m$-site EFP with $m>2$.

- In this work we demonstrated that the GGE is valid in the $q$-boson model. We argued that it holds for arbitrary $q$ and have rigorously proven it in the $q \rightarrow \infty$ limit in two special cases. The essential points were that the model has one particle species, all charges have finite values, and the GETH could be proven directly.

One of the most interesting open questions is whether there are other models where the GGE (built on the local charges only) gives a correct description of the stationary states.

We hope to return to these questions in further research.

\section{Acknowledgements}

We are grateful to Gábor Takács and Márton Kormos for useful discussions and for comments on the manuscript. In particular we are thankful to Márton Kormos for drawing our attention to the $q$-boson model, and to Gábor Takács for a fruitful discussion about the symmetric polynomials relevant to the present work. 


\section{References}

[1] A. Polkovnikov, K. Sengupta, A. Silva, and M. Vengalattore, "Colloquium : Nonequilibrium dynamics of closed interacting quantum systems," Rev. Mod. Phys. 83 (2011) 863-883.

[2] R. Nandkishore and D. A. Huse, "Many body localization and thermalization in quantum statistical mechanics," ArXiv e-prints (2014), arXiv:1404.0686 [cond-mat.stat-mech].

[3] I. Bloch, J. Dalibard, and S. Nascimbène, "Quantum simulations with ultracold quantum gases," Nat. Phys. 8 (2012) 267.

[4] M. Rigol, V. Dunjko, V. Yurovsky, and M. Olshanii, "Relaxation in a Completely Integrable Many-Body Quantum System: An Ab Initio Study of the Dynamics of the Highly Excited States of 1D Lattice Hard-Core Bosons," Physical Review Letters 98 (2007) no. 5, 050405, arXiv: cond-mat/0604476.

[5] M. Rigol, A. Muramatsu, and M. Olshanii, "Hard-core bosons on optical superlattices: Dynamics and relaxation in the superfluid and insulating regimes," Phys. Rev. A 74 (2006) 053616, arXiv: cond-mat/0612415.

[6] A. C. Cassidy, C. W. Clark, and M. Rigol, "Generalized Thermalization in an Integrable Lattice System," Physical Review Letters 106 (2011) 140405, arXiv: 1008.4794 [cond-mat.stat-mech].

[7] C. Gramsch and M. Rigol, "Quenches in a quasidisordered integrable lattice system: Dynamics and statistical description of observables after relaxation," Phys. Rev. A $\mathbf{8 6}$ (2012) 053615, arXiv:1206.3570 [cond-mat.stat-mech].

[8] T. M. Wright, M. Rigol, M. J. Davis, and K. V. Kheruntsyan, "Nonequilibrium Dynamics of One-Dimensional Hard-Core Anyons Following a Quench: Complete Relaxation of One-Body Observables," Phys. Rev. Lett. 113 (2014) 050601, arXiv:1312.4657 [cond-mat.quant-gas].

[9] T. Barthel and U. Schollwöck, "Dephasing and the Steady State in Quantum Many-Particle Systems," Physical Review Letters 100 (2008) no. 10, 100601, arXiv:0711.4896 [cond-mat.stat-mech].

[10] M. Kollar and M. Eckstein, "Relaxation of a one-dimensional Mott insulator after an interaction quench," Physical Review A 78 (2008) no. 1, 013626, arXiv:0804.2254 [cond-mat.str-el].

[11] M. A. Cazalilla, A. Iucci, and M.-C. Chung, "Thermalization and quantum correlations in exactly solvable models," Physical Review E $8 \mathbf{5}$ (2012) no. 1, 011133, arXiv:1106.5206 [cond-mat.stat-mech].

[12] A. Iucci and M. A. Cazalilla, "Quantum quench dynamics of the Luttinger model," Physical Review A 80 (2009) no. 6, 063619, arXiv:0903.1205 [cond-mat.str-el].

[13] P. Calabrese, F. H. L. Essler, and M. Fagotti, "Quantum quench in the transverse field Ising chain: I. Time evolution of order parameter correlators," Journal of Statistica Mechanics: Theory and Experiment 7 (2012) 16, arXiv:1204.3911 [cond-mat.quant-gas].

[14] P. Calabrese, F. H. L. Essler, and M. Fagotti, "Quantum quenches in the transverse field Ising chain: II. Stationary state properties," Journal of Statistical Mechanics. Theory and Experiment 7 (2012) 22, arXiv:1205.2211 [cond-mat.stat-mech].

[15] M. Fagotti and F. H. L. Essler, "Reduced Density Matrix after a Quantum Quench," Physical Review B 87 (2013) no. 24, 245107, arXiv:1302.6944 [cond-mat.stat-mech].

[16] S. Sotiriadis and P. Calabrese, "Validity of the GGE for quantum quenches from interacting to noninteracting models," Journal of Statistical Mechanics: Theory and Experiment 2014 (2014) no. 7, P07024, arXiv:1403.7431 [cond-mat.stat-mech].

[17] B. Wouters, M. Brockmann, J. De Nardis, D. Fioretto, and J.-S. Caux, "From Néel to XXZ: exact solution from the quench action," ArXiv e-prints (2014), arXiv:1405.0172 [cond-mat.str-el]. 
[18] J.-S. Caux and F. H. L. Essler, "Time Evolution of Local Observables After Quenching to an Integrable Model," Physical Review Letters 110 (2013) no. 25, 257203, arXiv:1301.3806 [cond-mat.stat-mech].

[19] B. Pozsgay, M. Mestyán, M. A. Werner, M. Kormos, G. Zaránd, and G. Takács, "Correlations after quantum quenches in the XXZ spin chain: Failure of the Generalized Gibbs Ensemble," ArXiv e-prints (2014), arXiv:1405.2843 [cond-mat.stat-mech].

[20] B. Pozsgay, "The generalized Gibbs ensemble for Heisenberg spin chains," Journal of Statistical Mechanics: Theory and Experiment 2013 (2013) no. 07, 3, arXiv:1304.5374 [cond-mat.stat-mech].

[21] M. Fagotti and F. H. L. Essler, "Stationary behaviour of observables after a quantum quench in the spin-1/2 Heisenberg XXZ chain," Journal of Statistical Mechanics. Theory and Experiment 7 (2013) 12, arXiv:1305.0468 [cond-mat.stat-mech].

[22] M. Fagotti, M. Collura, F. H. L. Essler, and P. Calabrese, "Relaxation after quantum quenches in the spin- $\frac{1}{2}$ Heisenberg XXZ chain," Physical Review B 89 (2014) 125101.

[23] B. Pozsgay, "Failure of the Generalized Eigenstate Thermalization Hypothesis in integrable models with multiple particle species," ArXiv e-prints (2014), arXiv: 1406.4613 [cond-mat.stat-mech].

[24] G. Goldstein and N. Andrei, "Failure of the GGE hypothesis for integrable models with bound states," ArXiv e-prints (2014), arXiv:1405.4224 [cond-mat.quant-gas].

[25] E. H. Lieb and W. Liniger, "Exact Analysis of an Interacting Bose Gas. I. The General Solution and the Ground State," Physical Review 130 (1963) 1605.

[26] J. Mossel and J.-S. Caux, "Exact time evolution of space- and time-dependent correlation functions after an interaction quench in the one-dimensional Bose gas," New Journal of Physics 14 (2012) no. 7, 075006, arXiv:1201.1885 [cond-mat.stat-mech].

[27] J.-S. Caux and R. M. Konik, "Constructing the Generalized Gibbs Ensemble after a Quantum Quench," Physical Review Letters 109 (2012) no. 17, 175301, arXiv:1203.0901 [cond-mat.quant-gas].

[28] D. Iyer, H. Guan, and N. Andrei, "Exact formalism for the quench dynamics of integrable models," Physical Review A 87 (2013) no. 5, 053628, arXiv:1304.0506 [cond-mat.quant-gas].

[29] M. Kormos, A. Shashi, Y.-Z. Chou, J.-S. Caux, and A. Imambekov, "Interaction quenches in the one-dimensional Bose gas," Physical Review B 88 (2013) no. 20. 205131, arXiv:1305.7202 [cond-mat.stat-mech].

[30] M. Kormos, M. Collura, and P. Calabrese, "Analytic results for a quantum quench from free to hard-core one dimensional bosons," Physical Review A 89 (2014) 013609, arXiv:1307.2142 [cond-mat.quant-gas].

[31] J. De Nardis, B. Wouters, M. Brockmann, and J.-S. Caux, "Solution for an interaction quench in the Lieb-Liniger Bose gas," Physical Review A 89 (2014) 033601, arXiv:1308.4310 [cond-mat.stat-mech].

[32] G. Goldstein and N. Andrei, "Equilibration and Generalized GGE in the Lieb Liniger gas," ArXiv e-prints (2013), arXiv:1309.3471 [cond-mat.quant-gas].

[33] G. Goldstein and N. Andrei, "How to experimentally detect a GGE? - Universal Spectroscopic Signatures of the GGE in the Tonks gas," ArXiv e-prints (2014), arXiv:1405.6365 [cond-mat.quant-gas].

[34] G. Goldstein and N. Andrei, "Quench between a Mott insulator and a Lieb-Liniger liquid," ArXiv e-prints (2014), arXiv:1406.4902 [cond-mat.quant-gas].

[35] N. M. Bogoliubov and R. K. Bullough, "A q-deformed completely integrable Bose gas model," Journal of Physics A: Mathematical and General 25 (1992) no. 14, 4057.

[36] N. Bogoliubov and R. Bullough, "Completely integrable model of interacting q-bosons," Physics Letters A 168 (1992) no. 4, 264-269. 
[37] N. M. Bogoliubov, R. K. Bullough, and G. D. Pang, "Exact solution of a q-boson hopping model," Physical Review B 47 (1993) 11495-11498.

[38] P. P. Kulish and E. V. Damaskinsky, "On the q oscillator and the quantum algebra su $(1,1), "$ Journal of Physics A: Mathematical and General 23 (1990) no. 9, L415.

[39] V. V. Cheianov, H. Smith, and M. B. Zvonarev, "Three-body local correlation function in the Lieb Liniger model: bosonization approach," J. Stat. Mech. 8 (2006) P08015, arXiv: cond-mat/0602468.

[40] N. Tsilevich, "Quantum inverse scattering method for the q-boson model and symmetric functions," Functional Analysis and Its Applications 40 (2006) no. 3. 207-217.

[41] F. Göhmann and V. E. Korepin, "Solution of the quantum inverse problem," J. Phys. A 33 (2000) no. 6, 1199 , arXiv: hep-th/9910253.

[42] J. M. Maillet and V. Terras, "On the quantum inverse scattering problem," Nuclean Physics B 575 (2000) 627-644, hep-th/9911030.

[43] B. Blass, H. Rieger, and F. Iglói, "Quantum relaxation and finite-size effects in the XY chain in a transverse field after global quenches," EPL (Europhysics Letters) 99 (2012) 30004, arXiv:1205.3303 [cond-mat.stat-mech].

[44] F. H. L. Essler, S. Evangelisti, and M. Fagotti, "Dynamical Correlations After a Quantum Quench," Physical Review Letters 109 (2012) no. 24, 247206, arXiv:1208.1961 [cond-mat.stat-mech].

[45] J. Mossel and J.-S. Caux, "Generalized TBA and generalized Gibbs," Journal of Physics A Mathematical General 45 (2012) no. 25, 255001, arXiv:1203.1305 [cond-mat.quant-gas].

[46] R. P. Feynman, "Forces in Molecules," Physical Review 56 (1939) 340.

[47] M. Mestyán and B. Pozsgay, "Short distance correlators in the XXZ spin chain for arbitrary string distributions," ArXiv e-prints (2014), arXiv:1405.0232 [cond-mat.stat-mech].

[48] S. Sorg, L. Vidmar, L. Pollet, and F. Heidrich-Meisner, "Relaxation and thermalization in the one-dimensional Bose-Hubbard model: A case study for the interaction quantum quench from the atomic limit," ArXiv e-prints (2014), arXiv:1405.5404 [cond-mat.stat-mech].

[49] N. M. Bogoliubov, "Boxed plane partitions as an exactly solvable boson model," Journal of Physics A: Mathematical and General 38 (2005) no. 43, 9415.

[50] N. Bogoliubov, "Enumeration of plane partitions and the algebraic Bethe anzatz," Theoretical and Mathematical Physics 150 (2007) no. 2, 165-174.

[51] N. Bogoliubov and J. Timonen, "Correlation functions for a strongly coupled boson system and plane partitions," Royal Society of London Philosophical Transactions Series A 369 (2011) 1319-1333.

[52] K. Shigechi and M. Uchiyama, "Boxed skew plane partition and integrable phase model," Journal of Physics A: Mathematical and General 38 (2005) no. 48, 10287.

[53] N. Bogoliubov, A. Izergin, and N. Kitanine, "Correlation functions for a strongly correlated boson system," Nuclear Physics B 516 (1998) no. 3, 501 - 528.

[54] P. Barmettler, M. Punk, V. Gritsev, E. Demler, and E. Altman, "Quantum quenches in the anisotropic spin-1/2 Heisenberg chain: different approaches to many-body dynamics far from equilibrium," New Journal of Physics 12 (2010) no. 5, 055017, arXiv:0911.1927 [cond-mat.quant-gas].

[55] M. Brockmann, J. De Nardis, B. Wouters, and J.-S. Caux, "A Gaudin-like determinant for overlaps of Néel and XXZ Bethe states," Journal of Physics A Mathematical General 47 (2014) no. 14, 145003, arXiv:1401.2877 [cond-mat.stat-mech]).

[56] M. Brockmann, J. De Nardis, B. Wouters, and J.-S. Caux, "Néel-XXZ state overlaps: odd particle numbers and Lieb-Liniger scaling limit," ArXiv e-prints (2014), arXiv:1403.7469 [cond-mat.stat-mech]. 